\title{
Biologically Active Volatile Organic Compounds (VOCs) Produced by Rhizospheric Actinobacteria Strains Inhibit the Growth of the Phytopathogen Colletotrichum musae
}

\author{
Maria V. de Brito, ${ }^{\circledR a}$ Wéverson L. Fonseca, ${ }^{b}$ Jair Mafezoli, ${ }^{a}$ Francisco G. \\ Barbosa, ${ }^{a}$ Fátima M. Nunes, ${ }^{a}$ Marcos C. de Mattos, ${ }^{\circledR a}$ João E. A. dos Santos, ${ }^{a}$ \\ Francisca S. A. Araujo, ${ }^{c}$ Regimara F. B. S. Vieira, ${ }^{a}$ Hilton C. R. Magalhães, ${ }^{c}$

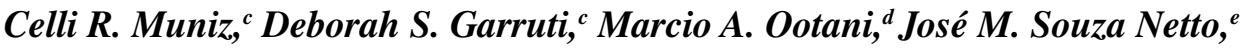 \\ Licarion Pinto, ${ }^{\oplus e}$ Francisco M. P. Viana ${ }^{\dagger, c}$ and Maria C. F. Oliveira ${ }^{\circledR *, a}$ \\ ${ }^{a}$ Departamento de Química Orgânica e Inorgânica, Centro de Ciências, \\ Universidade Federal do Ceará, Campus do Pici, Bl. 940, 60440-593 Fortaleza-CE, Brazil \\ ${ }^{b}$ Colégio Técnico de Bom Jesus (CTBJ), Universidade Federal do Piauí, BR 135, km 3, \\ Planalto Horizonte, 64900-000 Bom Jesus-PI, Brazil
}

${ }^{c}$ EMBRAPA Agroindústria Tropical, Rua Pernambuco 2270, Pici, 60511-110 Fortaleza-CE, Brazil

${ }^{d}$ Centro de Tecnologias Estrategicas do Nordeste (CETENE), Av. Prof. Luiz Freire, Cidade Universitária, 50740-540 Recife-PE, Brazil

${ }^{e}$ Laboratório de Metabolômica e Quimiometria, Universidade Federal de Pernambuco, Av. Jornalista Anibal Fernandes s/n, Cidade Universitária, 50740-560 Recife-PE, Brazil

The antifungal potential of volatile organic compounds (VOCs) produced by actinobacterial strains Streptomyces sp. (ACTB-77) and Amycolatopsis sp. (ACTB-290) from the rhizosphere of Caatinga plants against Colletotrichum musae was investigated. VOCs produced by these microorganisms (axenic and co-culture) were investigated using headspace-solid phase microextraction (HS-SPME) and gas chromatography-mass spectrometry (GC-MS). Although no exclusive VOC peaks were observed in the co-culture with ACTB-77, the same experiment involving ACTB-290 yielded five new peaks, including two identified alcohols, suggested as bioreductive products of the corresponding ketones by the fungus. Statistical analysis revealed that co-culture ACTB-77/C. musae has a closer similarity to the fungus than to the actinobacteria, while the co-culture ACTB-290/C. musae showed closer similarity to the actinobacteria. These confirmed the more pronounced antifungal activity of the ACTB-290 strain, as observed in the fungus growth inhibition experiments. The antifungal activity of ACTB-290 was associated to its sulfur-containing metabolites, while linalool was suggested as responsible for the ACTB-77 activity.

Keywords: VOCs, HS-SPME-GCMS, Amycolatopsis, Streptomyces, Colletotrichum musae, antifungal

\section{Introduction}

Colletotrichum species are recognized as phytopathogenic fungi accountable for anthracnose diseases in a great variety of crops distributed worldwide. ${ }^{1}$ Among them, $C$. musae is known to be responsible for the main

\footnotetext{
*e-mail: mcfo@ufc.br

'In memoriam. This paper is dedicated to Dr Francisco Marto Pinto Viana, Brazilian phytopathologist who motivated us to develop this research and passed away during the article preparation.

Editor handled this article: Paulo Cezar Vieira
}

postharvest anthracnose in bananas. This quiescent fungus contaminates the fruit at the preharvest stage, and the disease symptoms emerge at postharvest when the fruit has achieved an advanced stage of maturity. ${ }^{2,3}$ The use of fungicides to control C. musae is considered an unfeasible approach because it demands multiple pulverizations. ${ }^{3}$ Moreover, consumers prefer fruit submitted to low pesticide. This has forced some countries to legislate stricter regulations on the maximum limits of pesticides in fruits for import and export. ${ }^{2}$ Therefore, the use of biocontrol agents (BCA) to substitute for chemical fungicides is a greener 
and more efficient strategy for controlling phytopathogens, including C. musae. ${ }^{1-3}$

Microorganisms present a sophisticated metabolism that is responsible for the production of a myriad of metabolites with diverse molecular structures. Many of the microbial produced compounds, especially those from the secondary metabolism, are known for their biotechnological potential. ${ }^{4,5}$ Besides producing non-volatile chemical structures, microorganisms can also provide volatile organic compounds (VOCs), also referred as microbial VOCs (mVOCs), which play an important role in a number of microbe-microbe interactions. ${ }^{6}$

Microbial VOCs are composed of lipophilic chemical structures with high vapor pressure, which pass through biological membranes (live organisms) and released into the environment where the living organism is found. ${ }^{7}$ Many of these compounds are by-products of primary metabolisms originating from different pathways, such as fermentation, aerobic heterotrophy, amino acid catabolism, sulfur reduction, and terpenoid biosynthesis. About 2,000 mVOCs have been identified, with the most common belonging to the chemical classes of alcohols, alkanes, alkenes, aromatic compounds, nitrogen- and sulfur-containing compounds, and terpenes. ${ }^{8}$

When subjected to co-culture techniques, microorganisms can activate silenced genes, producing mVOCs. ${ }^{9,10}$ that will play important role in various microbemicrobe interactions, including antagonism. This latter relationship has been used in studies aiming to identify the mVOCs responsible for performing inhibitory activity against phytopathogens. ${ }^{11,12}$ In this context, rhizosphere microorganisms, especially bacterial strains, play an important role in agriculture, protecting plants against phytopathogens, ${ }^{13}$ and being used as biological control agents in the effective management of plant diseases. ${ }^{14}$ These actinobacteria (filamentous Gram-positive bacteria) are important as rich sources of secondary metabolites, ${ }^{15,16}$ being responsible for the production of various VOCs.

Actinobacteria have great potential of VOCs production, with the most frequently identified compounds being butan-1-ol, 2-methylpropan-1-ol, 3-methylbut-3-en1-ol, 3-methylbutan-1-ol, dimethyl disulfide, dimethyl trisulfide, 2-phenylethanol and geosmin. ${ }^{16,17}$ This latter $\mathrm{mVOC}$ is a sesquiterpene responsible for the Petrichor, or "after-the-rain", smell. Streptomyces species are responsible for producing many VOCs, most of them derived from terpenoids with antifungal properties and biocontrol effectiveness. For example, methylvinylketone, produced by $S$. griseoruber, is reported to inhibit the germination of spores from the fungus Cladosporium cladosporioides. ${ }^{18}$ Likewise, VOCs produced by S. alboflavus and S. philanthi promoted growth inhibition of Fusarium moniliforme,
F. fujikuroi, Aspergillus flavus, A. ochraceus, A. niger, Penicillium citrinum, Rhizoctonia solani, Pyricularia grisea and Bipolaris oryzae. ${ }^{19}$ Additionally, VOCs from S. globisporus inhibited the mycelial growth, spore germination and appressoria formation of Botrytis cinerea in tomatoes and protected them against the post-harvest gray mold caused by this fungus. ${ }^{20}$

Studies on mVOCs highlight the use of headspace-solid phase micro-extraction (HS-SPME) as appropriate tool to obtain the compounds under relatively mild conditions. The extraction occurs in the absence of solvent, and it is based on the partition equilibrium of analytes between the sample matrix and the extraction phase. ${ }^{21,22}$ Different types of mixed fibers can be used as matrix, including polydimethylsiloxane-divinylbenzene-carboxen (PDMS/DVB/CAR), polydimethylsiloxane-divinylbenzene (PDMS/DVB) and polydimethylsiloxane-carboxen (PDMS/CAR). Therefore, an effective extraction of VOCs using HS-SPME requires the optimization of the protocol, varying important parameters, such as fiber coating and headspace conditions (e.g., extraction time and temperature). ${ }^{23}$ After the HS-SPME procedure, the separation and identification of the extracted compounds is carried out through the hyphenated technique of gas chromatography-mass spectrometry (GC-MS). ${ }^{24,25}$

Comparative studies of mVOCs produced by actinobacteria strains from rhizosphere cultured under axenic and co-culture conditions to identify antifungal compounds are still incipient. In the present paper, we report the results from the mVOCs produced by twenty actinobacteria strains, isolated from rhizosphere associated with Caatinga growing plants, with antagonist effect against the phytopathogen fungus Colletotrichum musae. The Caatinga is a semi-arid ecosystem found exclusively in Brazil. Because Amycolatopsis sp. (ACTB-290) and Streptomyces sp. (ACTB-77) were the most promising strains against Colletotrichum musae, the VOCs produced by these microorganisms under axenic and co-culture conditions were investigated.

\section{Experimental}

\section{Chemicals}

Potato-dextrose-agar (PDA) culture medium was purchased from Kasvi ${ }^{\circledR}$ (Campinas, Brazil). All other chemicals were from Sigma-Aldrich ${ }^{\circledR}$ (São Paulo, Brazil).

\section{Microorganisms and culture medium}

Twenty strains of actinobacteria from the rhizosphere of Caatinga plants (Table 1) used in this work were obtained 
from the Laboratory of Phytopathology at Embrapa Tropical Agroindustry (CNPAT, Fortaleza, Ceará, Brazil).

Table 1. Actinobacteria strains isolated from Caatinga plants and their respective geographic locations

\begin{tabular}{|c|c|c|}
\hline Strain code & Plant source & Geographic location \\
\hline ACTB-10 & Mimosa arenosa & \\
\hline ACTB-25 & M. arenosa & $4^{\circ} 11^{\prime} 26.62^{\prime \prime} \mathrm{S} 38^{\circ} 29^{\prime} 50.78^{\prime \prime} \mathrm{W}$ \\
\hline ACTB-32 & Bauhinia forficata & \\
\hline ACTB-77 & Combretum leprosum & 66'1.18”'S 39॰23'0.29”'W \\
\hline ACTB-166 & Sideroxylon obtusifolium & $4^{\circ} 33^{\prime} 4.44^{\prime \prime} \mathrm{S} 39^{\circ} 37^{\prime} 53.18^{\prime \prime} \mathrm{W}$ \\
\hline ACTB-171 & Croton blanchetianus & $5^{\circ} 12^{\prime} 55.97^{\prime \prime} \mathrm{S} 37^{\circ} 0{ }^{\prime} 57.07^{\prime \prime} \mathrm{W}$ \\
\hline ACTB-172 & Myracrodruon urundeuva & \\
\hline ACTB-177 & Cordia trichotoma & \\
\hline АCTB-227 & C. trichotoma & \\
\hline АCTB-242 & Cereus jamacaru & \\
\hline АСТВ-246 & C. jamacaru & \\
\hline АCTB-282 & Commiphora leptophloeos & \\
\hline ACTB-285 & Anadenanthera colubrina & $4^{\circ} 33^{\prime} 4.44^{\prime \prime} \mathrm{S} 39^{\circ} 37^{\prime} 53.18^{\prime \prime} \mathrm{W}$ \\
\hline АCTB-286 & A. colubrina & \\
\hline АCТВ-290 & A. colubrina & \\
\hline АСТВ-291 & A. colubrina & \\
\hline АСТВ-292 & A. colubrina & \\
\hline АCTB-296 & A. colubrina & \\
\hline АCTB-299 & Croton blanchetianus & \\
\hline ACTB-305 & Licania rigida & $4^{\circ} 33^{\prime} 7.98^{\prime \prime} \mathrm{S} 99^{\circ} 38^{\prime} 24.48^{\prime \prime} \mathrm{W}$ \\
\hline
\end{tabular}

S: south; W: west.

The strain of the phytopathogenic fungus Colletotrichum musae (MMBF226/12) was donated by the Biological Institute of São Paulo. C. brevisporum (CMM-1179), Lasiodiplodia brasiliense (strains CMM-2248 and CMM-2253), L. theobramae (CMM-22004), L. hormozganensis (CMM-2211) and L. viticola (CMM-2252) strains were from the Federal Rural University of Pernambuco (UFRPE), collection of phytopathogenic fungi culture-Prof Maria Menezes (CMM collection).

All microorganisms were cultured in commercially available potato dextrose agar medium $\left(39.0 \mathrm{~g} \mathrm{~L}^{-1}\right)$, consisting of $84.4 \%$ of potato broth, $8.4 \%$ of dextrose and $7.2 \%$ of bacteriological agar.

\section{Screening on actinobacteria producing VOCs with antifungal activity against $C$. musae}

The selection of VOCs-emitting actinobacteria strains with antifungal activity followed the methodology described in literature ${ }^{26}$ with $C$. musae used as target fungus. The 20 strains of actinobacteria were grown, separately in PDA for 5 days. Subsequently, a $5 \mathrm{~mm}$ disk of the culture of each of these microorganisms was transferred to one side of a bi-Petri plate (Figure S1, Supplementary Information (SI) section) and the plate was kept for 2 days under a static condition at $28{ }^{\circ} \mathrm{C}$. After that, a $5 \mathrm{~mm}$ disk of mycelium of the C. musae, previously grown in PDA for 5 days, was inoculated on the other side of the bi-Petri plate (Figure S1). For each experiment, control plates were prepared containing only the C. musae (absence of actinobacteria). All the plates were wrapped with double layers of parafilm and incubated under static conditions at $28{ }^{\circ} \mathrm{C}$ for 5 days. The inhibition of mycelial growth of the fungus was expressed in percentage, equation 1 , considering the average diameters of the fungus growth in the control plate and in the experiment. ${ }^{27}$ The diameters were measured with a $100 \mathrm{~mm}$ electronic digital caliper ruler carbon fiber composite vernier. Bioassays were performed in triplicate and the data obtained were analyzed according to one-way analysis of variance (ANOVA); the values were considered significant when $p<0.05$ (GraphPad Prism) ${ }^{28}$

$\mathrm{GI}(\%)=\frac{\varnothing \mathrm{c}-\varnothing \mathrm{e}}{\varnothing \mathrm{c}} \times 100$

where Øc and Øe represent the average diameters of the growth fungus in the Petri dishes from the control (fungus only) and experimental sample with the actinobacteria, respectively, and GI is growth inhibition.

Antifungal activity of VOCs produced by ACTB-290 and ACTB-77 strains against $C$. musae

The antifungal activity of VOCs emitted by actinobacteria Amycolatopsis sp. (ACTB-290) and Streptomyces sp. (ACTB-77) was assayed against the phytopathogenic fungus $C$. musae using Petri dish ( $80 \mathrm{~mm}$ diameter) and following the double-dishes method. ${ }^{29}$ A cell suspension $(10 \mu \mathrm{L})$ of the actinobacteria was inoculated in a Petri dish containing PDA culture medium. The plate was incubated for $48 \mathrm{~h}$, at $28^{\circ} \mathrm{C}$, under static condition. After this period, a $5 \mathrm{~mm}$ disk of the previously cultured fungus mycelium (5 days old) was inoculated in the center of another $80 \mathrm{~mm}$ diameter Petri dish containing PDA (Figure S2, SI section). Then, the Petri dishes containing the microorganisms (actinobacteria and fungus) were placed inversely over each other without the lids to establish a double dish set, and immediately sealed with parafilm. In this case, the distance between the micro-organisms cultures was $1.5 \mathrm{~cm}$ (Figure S2). The double dish sets were set up in such a manner that the fungus and actinobacteria cultures were placed opposite each other, with the fungus on top and the 
actinobacteria at the bottom. The experiment was incubated under static conditions at $28^{\circ} \mathrm{C}$ for 5 days. As the control experiment, Petri dishes were inoculated with the fungus exposed to the PDA culture medium only. The diameter $(\mathrm{mm})$ of the fungus mycelium was measured daily until the fungus finished growing on the fifth day in the control experiment. Experiments were carried out in triplicate and all data obtained, using the computer GraphPad Prism program, ${ }^{28}$ were analyzed according to one-way ANOVA, which determined a significant difference with $p<0.05$.

Antifungal activity of VOCs produced by ACTB-290 strain against other phytopathogen fungi

The antifungal activity of VOCs emitted by actinobacteria Amycolatopsis sp. (ACTB-290) was assayed against the phytopathogenic fungal strains C. brevisporum, Lasiodiplodia brasiliense, L. theobramae, L. hormozganensis, L. brasiliense and L. viticola, following the same procedure described above for C. musae. In this case, the diameter ( $\mathrm{mm}$ ) of each mycelium was measured daily until the fungus finished growing in the control experiment, which varied as follows: $C$. brevisporum (8 days), L. theobramae (8 days), L. brasiliense (strain CMM-2248: 5 days; strain CMM-2253: 4 days), L. hormozganensis (4 days) and L. viticola (3 days).

Molecular identification of actinobacteria strains ACTB-290 and ACTB-77

The genomic deoxyribonucleic acid (DNA) of all strains was extracted using the Bacterial Genomic DNA purification kit from HIMEDIA (Mumbai, India), following the manufacturer's instructions. DNA quantification was performed by the NanoDrop ${ }^{\circledR}$ 2000c spectrophotometer (Thermo Fisher Scientific, Massachusetts, USA), version 1.0 , and the concentration of $10 \mathrm{ng} \mu \mathrm{L}^{-1}$ was then diluted and stored at $-20^{\circ} \mathrm{C}$. The nucleotide sequence of the $16 \mathrm{~S}$ genomic region of ribosomal DNA (rDNA) was amplified by a polymerase chain reaction (PCR) using primers 27F (5'-GAGTTTGATCMTGGCTCAG-3') and 1492R (5'-ACGGYTACCTTGTTACGACTT-3'). The PCR mixtures $(50 \mu \mathrm{L})$ contained $6.25 \mu \mathrm{L}$ of genomic DNA (10 ng $\left.\mu \mathrm{L}^{-1}\right), 10 \mu \mathrm{L}$ of $5 \times$ buffer, $1 \mu \mathrm{L}$ of deoxynucleotide triphosphate (dNTP) $(10 \mathrm{mM}), 2 \mu \mathrm{L}$ of $\mathrm{MgCl}_{2}(25 \mathrm{mM})$, $0.8 \mu \mathrm{L}$ of each primer $(10 \mathrm{mM}), 0.5 \mu \mathrm{L}$ of GoTaq polymerase $\left(5 \mathrm{U}_{\mu} \mathrm{L}^{-1}\right)$ and $28.65 \mu \mathrm{L}$ of ultrapure sterile water.

A Flexigene thermal cycler from Techne (Woonsocket, USA) was used in the PCR amplifications, programed as follows: initial denaturation step at $94{ }^{\circ} \mathrm{C}$ for $2 \mathrm{~min}$, followed by 35 denaturation cycles at $94{ }^{\circ} \mathrm{C}$ for $60 \mathrm{~s}$, annealing at
$56{ }^{\circ} \mathrm{C}$ for $45 \mathrm{~s}$ and extension $72{ }^{\circ} \mathrm{C}$ for $60 \mathrm{~s}$, with a final extension at $72{ }^{\circ} \mathrm{C}$ for $10 \mathrm{~min} .{ }^{30}$ The PCR products were separated by electrophoresis on $1.5 \%$ agarose gel in $1 \mathrm{X}$ Tris-borate-ethylenediaminetetraacetic acid (EDTA) buffer, stained with ethidium bromide $\left(0.5 \mathrm{mg} \mathrm{mL}^{-1}\right)$ for $1 \mathrm{~min}$ and visualized under UV. After checking the amplified bands, $40 \mu \mathrm{L}$ aliquots of each PCR product were purified and sequenced by Macrogen Inc. (Seoul, South Korea).

The nucleotide sequences were edited using the BioEdit program version 7.0.5 $5^{31}$ and were subjected to identity verification using the basic local alignment search tool (Basic Local AlignmentSearch Tool = BLASTn) from GenBank, ${ }^{32}$ followed by manually alignment using ClustalW ${ }^{33}$ with strings previously published and deposited in GenBank (NCBI). The phylogenetic analyses of maximum parsimony were performed using the PAUP 4.0 program beta $10 .{ }^{34}$ For the analysis of maximum parsimony, the following options were selected: heuristic searches, tree-bisection-reconnection (TBR), branch swapping and MULTREES. The statistical support of the tree was tested using bootstrap analysis with 1,000 replicates.

\section{Scanning electron microscope (SEM) imaging of C. musae} hyphae

Morphological analyses of $C$. musae was performed in a scanning electron microscope (SEM), model $940 \mathrm{~A}$, from Zeiss DSM (Jena, Germany), at an acceleration voltage of $15 \mathrm{kV}$. Samples (1-5 mm cubes) of PDA containing the fungus hyphae were transferred to a $2 \mathrm{~mL}$ Eppendorf vial already containing $1 \mathrm{~mL}$ of Karnovsky solution. ${ }^{35}$ After $1 \mathrm{~h}$ at room temperature under static conditions, the Karnovsky solution was removed, and samples were washed for $10 \mathrm{~min}$ with $0.1 \mathrm{M}$ phosphate buffer solution $(3 \times 2 \mathrm{~mL})$. An aliquot (enough to cover the samples) of $1 \%$ solution of $\mathrm{OsO}_{4}$ was added to the Eppendorf vial and maintained for $1 \mathrm{~h}$ at room temperature under static condition. After removing the solution, the samples were washed three times (15 min between each washing) with distilled water, followed by dehydration with increasing concentration of ethanol solution (20, 40, 60, 80 and 100\%). Subsequently, the samples were brought to the critical drying point in a critical point drying apparatus, model K850, from Quorum Technologies (Laughton, England), then placed in metallic sample holders (stabs). After being coated with a gold layer, the samples were analyzed in SEM equipment.

\section{Optimization of mVOCs extraction by HS-SPME}

The experiment to optimize mVOCs extraction by HS-SPME was carried out with the actinobacteria 
Amycolatopsis sp. (ACTB-290) and it was based on a similar experiment described in the literature. ${ }^{36}$ The following Supelco $^{\circledR}$ (Pennsylvania, USA) solid phase microextraction fibers (SPME), needle size 24ga and fiber length $1 \mathrm{~cm}$, were used: polydimethylsiloxane-divinylbenzene-carboxen (PDMS/DVB/CAR; fiber diameter $\left(\mathrm{d}_{\mathrm{f}}\right): 50 / 30 \mathrm{~mm}$ ), polydimethylsiloxane-divinylbenzene (PDMS/DVB; $\mathrm{d}_{\mathrm{f}}: 65 \mathrm{~mm}$ ) and polydimethylsiloxane-carboxen (PDMS/ CAR; $\mathrm{d}_{\mathrm{f}}: 85 \mathrm{~mm}$ ). The fibers were conditioned prior to use following the manufacturer's recommendations.

The actinobacteria strain was inoculated into Petri dishes containing PDA medium and incubated for 7 days at $28{ }^{\circ} \mathrm{C}$ under static condition. Subsequently, $10 \mathrm{~mL}$ of the still liquid PDA medium was added to a $20 \mathrm{~mL}$ vial. After solidification of the medium in the vial, a $5 \mathrm{~mm}$ disk of the previously inoculated actinobacteria was added. The vial containing the inoculum was immediately closed with silicone septum, closed with a threaded cap and maintained 5 days at $28{ }^{\circ} \mathrm{C}$ under static condition. Then, the vial was placed in a water bath (at 30 or $50^{\circ} \mathrm{C}$ ) and, after $5 \mathrm{~min}$, a fiber was inserted through a hole in the septum $(1 \mathrm{~cm}$ above the microorganism), Figure S3, SI section. The time of VOCs extraction varied from 10 to $40 \mathrm{~min}$. After each extraction time, the fiber was removed from the vial, and inserted into a gas chromatograph flame ionization detector (GC-FID) injector model QP-2010s (Shimadzu Corporation, Tokyo, Japan) for $5 \mathrm{~min}$ at $250{ }^{\circ} \mathrm{C}$ (splitless mode) for thermal desorption of the analytes. The GC-FID was equipped with a DB-5MS capillary column (film thickness: $30 \mathrm{~m} \times 0.25 \mathrm{~mm} \times 0.25 \mu \mathrm{m}$ ) from Agilent $\mathrm{J} \& \mathrm{~W}$ GC Columns (Santa Clara, USA). Analytical conditions were: GC oven temperature $40{ }^{\circ} \mathrm{C}$ for $2 \mathrm{~min} ; 10{ }^{\circ} \mathrm{C} \mathrm{min}{ }^{-1}$ up to $195{ }^{\circ} \mathrm{C} ; 7{ }^{\circ} \mathrm{C} \mathrm{min}^{-1}$ up to $220{ }^{\circ} \mathrm{C} ; 10{ }^{\circ} \mathrm{C} \min ^{-1}$ up to $260{ }^{\circ} \mathrm{C}$. Volumetric flow of the mobile phase (helium gas) of $0.59 \mathrm{~mL} \mathrm{~min}^{-1}$; detector temperature was $250{ }^{\circ} \mathrm{C}$. All experiments were carried out in triplicate, resulting in 72 analyses. Number of peaks and areas were presented as total mean values of all compounds. The obtained data were analyzed using the free software for statistical computing, R Program. ${ }^{37}$

HS-SPME and GC-MS analysis of VOCs produced by Amycolatopsis sp. (ACTB-290) and Streptomyces sp. (ACTB-77) under axenic culture and co-culture with C. musae

\section{mVOCs extraction by HS-SPME}

Experiments of VOCs extraction were performed with the actinobacteria Amycolatopsis sp. (ACTB-290) and Streptomyces sp. (ACTB-77) under axenic culture and co-cultured with $C$. musae. All microorganisms were previously grown, separately, in Petri dishes containing PDA medium for 5 days at $28{ }^{\circ} \mathrm{C}$ under static condition. Microbial VOCs extraction by HS-SPME was carried out using the optimized conditions (fiber: PDMS/DVB/CAR; extraction time: $30 \mathrm{~min}$; extraction temperature: $50{ }^{\circ} \mathrm{C}$ ).

Three $20 \mathrm{~mL}$ were used, two for axenic cultures (actinobacteria and fungus) and one for co-culture (actinobacteria together with fungus). PDA medium $(10 \mathrm{~mL})$ was added to each vial of axenic culture and the microorganisms (actinobacteria and fungus) were inoculated in the center of each vial. In the case of the coculture experiment, $3 \mathrm{~mL}$ of the culture medium was placed on one side of the glass container, which was in a horizontal position. After solidification of the PDA medium, an additional $3 \mathrm{~mL}$ of the medium was placed on the other side of the same vial (Figure S4, SI section). Initially, a $5 \mathrm{~mm}$ disk of each actinobacteria strain previously inoculated in the Petri dish was added to the vial corresponding to its individual culture experiment and another $5 \mathrm{~mm}$ disk to one side of the vial of the co-culture experiment. After $48 \mathrm{~h}$, a $5 \mathrm{~mm}$ disk of the fungus previously inoculated in the Petri dish was added to the flask corresponding to the individual culture experiment and another $5 \mathrm{~mm}$ disk to the other side of the flask of the co-culture experiment (opposite side of the actinobacteria strain).

After 5 days of inoculation of the microorganisms, all vials were placed in a water bath at $30^{\circ} \mathrm{C} ; 5$ min later, the PDMS/DVB/CAR fiber was inserted through a hole in the septum ( $1 \mathrm{~cm}$ above the microorganism). Microbial VOCs were extracted for $30 \mathrm{~min}$ at $30{ }^{\circ} \mathrm{C}$. Then, the fiber was removed and inserted into the GC-MS injector using the same conditions previously described in the experimental of optimization of mVOCs extraction by HS-SPME. The experiments were carried out in triplicate and PDA medium (without microorganism) was used as control, resulting in 18 analyses (3 axenic culture of ACTB-290; 3 axenic cultures of ACTB-77; 3 axenic cultures of C. musae; 3 co-cultures of ACTB-290 + C. musae; 3 co-cultures of ACTB-77 + C. musae 3 control).

\section{mVOCs analysis by GC-MS}

Extracted $\mathrm{mVOCs}$ were analyzed in a gas chromatograph (GC model 7890B) coupled to a mass spectrometer (MS model 5977A MSD) from Agilent Technologies Spain (Madrid, Spain). Compounds were separated in GC using the same conditions previously described in the experimental of optimization of mVOCs extraction by HS-SPME. Electron impact ( $70 \mathrm{eV}$ ) MS data were recorded with $\mathrm{m} / \mathrm{z}$ from 50 to 500 Daltons at intervals of $0.5 \mathrm{~s}$.

Compound identification was carried out by comparison of the mass spectra obtained for each compound with the 
one reported in mass spectral libraries,${ }^{38}$ including NIST 05 and NIST 27 (National Institute of Standards and Technology, Gaithersburg, USA), ${ }^{39}$ as well as PUBCHEM. ${ }^{40}$ Additionally, the Kovats index was calculated for each compound using a mixture of saturated $n$-alkanes C7-C30.

\section{Statistical analysis of the experiments}

The data matrices were used in the present work for pattern recognition statistical analysis after a pretreatment. This latter corresponds to the integrated peaks of the major compounds after removing all peaks of the control experiments (culture medium blank) and baseline (noise and low concentration peaks).

Matrix 1 refers to $C$. musae, ACTB-77 and their co-culture (C. musae and ACTB-77); matrix 2 refers to C. musae, ACTB-290 and their co-culture (C. musae and ACTB-290); matrix 3 refers all samples used in matrices 1 and 2. For all these matrices, lines correspond to these samples and columns correspond to the mVOCs peaks after the pretreatment. The organized matrices were autoscaled and then subjected to a principal component analysis (PCA) to observe differences and similarities between the samples. For matrix 1 and 2, two principal components (PC) were used while three PCs were used for matrix 3. PCA calculations were performed using PLS-ToolBox 5.2 $2^{41}$ and Matlab ${ }^{\circledR} 2010 .{ }^{42}$

The significant differences between each group were calculated with a $t$-test using the scores values of each individual class, similar to what was initially used in the SIMCA (Soft Independent Method of Class Analogy) models. ${ }^{43}$

\section{Results and Discussion}

Screening of actinobacteria producing VOCs with antifungal activity against $C$. musae

Twenty strains of actinobacteria isolated from rhizosphere of plants from the Caatinga biome (Table 1) were assayed for their ability to produce VOCs capable of inhibiting the growth of $C$. musae, a phytopathogen fungus responsible for causing anthracnosis in banana culture. ${ }^{2}$ Experiments were performed in bi-Petri dishes (Figure 1) and growth inhibition (GI) was recorded after 5 days of culturing. GI percentages were calculated through comparison between the control experiments (fungus only; GI 0\%) and experiments with both microorganisms (fungus and actinobacteria). Figure 1 shows that all actinobacteria strains assayed promoted inhibition of fungal growth (GI 50.3-73.7\%) and, among them, ACTB-77 (GI 68.0\%) and ACTB-290 (GI 73.7\%) promoted the highest inhibitory effect. Therefore, these two strains were selected for molecular identification and further VOCs investigation under axenic and co-culture conditions.

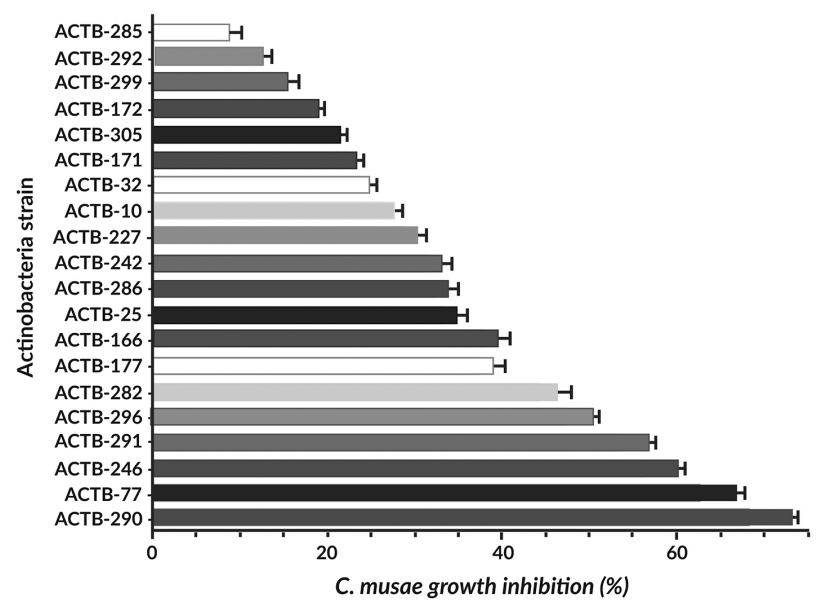

Figure 1. Growth inhibition (\%) of C. musae by volatile organic compounds (VOCs) produced by the twenty actinobacteria strains assayed, calculated through comparison of the control experiments (fungus only; GI 0\%) and experiments with both microorganisms (fungus and actinobacteria).

Molecular identification of strains ACTB-77 and ACTB-290

The promising antifungal potential activity of VOCs produced by strains ACTB-77 and ACTB-290 on the growth inhibition of $C$. musae motivated their identification by molecular approach. Strains ACTB-77 and ACTB-290 showed 99\% similarity to Streptomyces spp. and Amycolatopsis spp., respectively. According to the most parsimonious phylogenetic tree (Figure 2) obtained from the $16 \mathrm{~S}$ sequence data set of strains of the genus Amycolatopsis, the sequence of strain ACTB-290 was grouped in a clade with strains of Amycolatopsis sp. with $100 \%$ bootstrap support, strain while ACTB-77 was grouped in a distinct clade of the genus Streptomyces sp.

Antifungal activity of VOCs from Streptomyces sp. (ACTB-77) and Amycolatopsis sp. (ACTB-290) against C. musae

To evaluate the antifungal activity of VOCs produced by the two selected strains in more detail, a new experiment was carried out to monitor growth inhibition starting from the first day of microbial inoculation until the fifth day of the experiment when the control fungus strain occupied $100 \%$ of the Petri dish. As observed in Figure 3, the two strains promoted similar growth inhibitions of the fungus, which initiated from the second day of incubation and continued until the end of the experiment. 


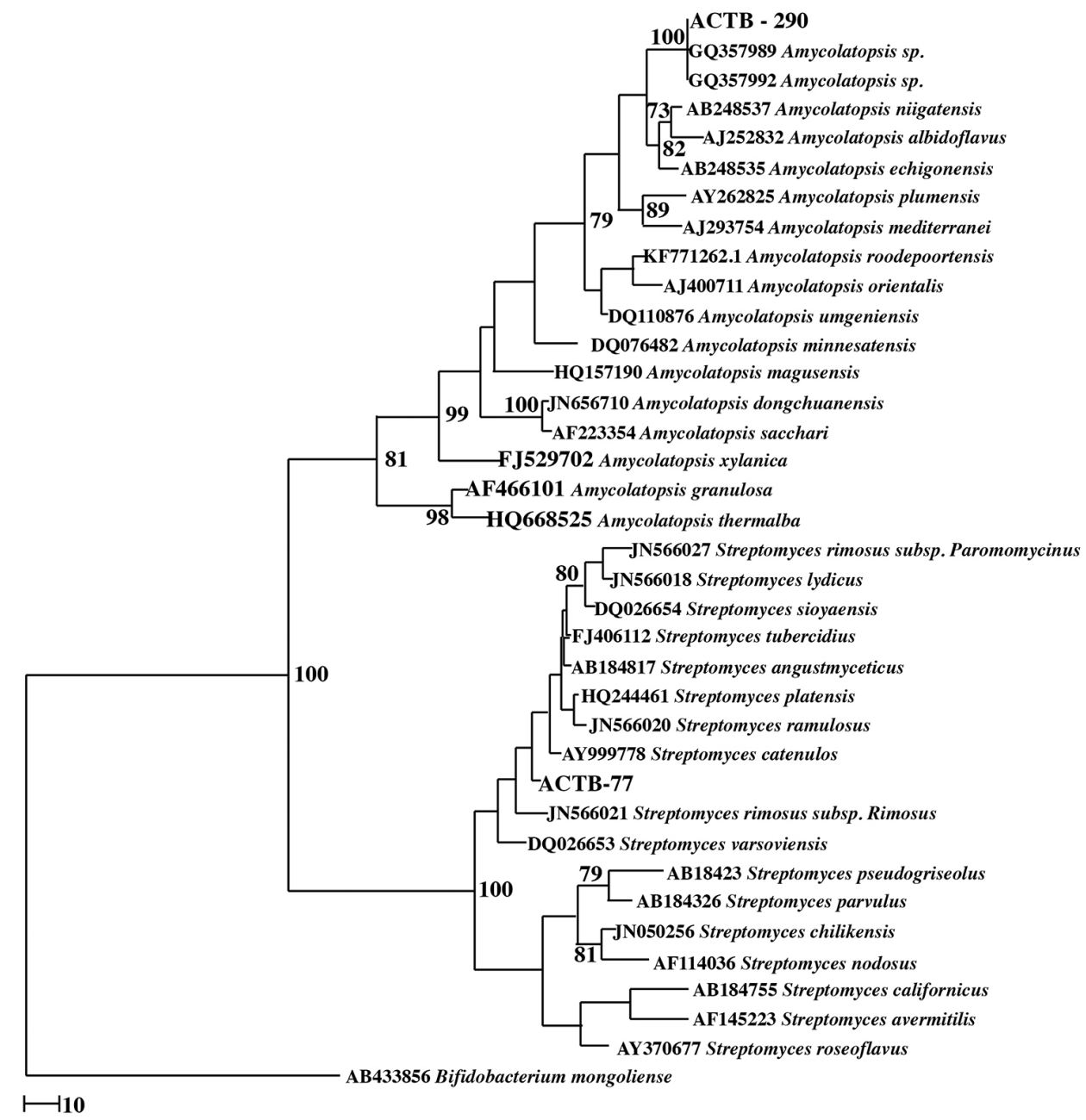

Figure 2. Phylogenetic tree inferred by maximum parsimony (MP) of data from the $16 \mathrm{~S}$ genomic region of the rDNA for sequences of the genera Amycolatopsis and Streptomyces. Bootstrap values (> 70\%) with 1000 repetitions are shown in the respective branch. Bifidobacterium mongoliense was used as an external group. The sequences in this study are highlighted in bold.

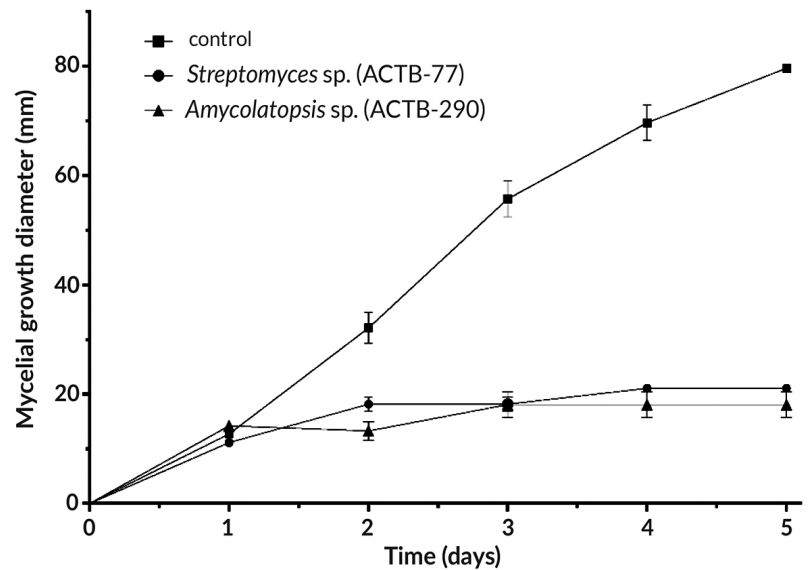

Figure 3. Results from the antifungal activity of Streptomyces sp. (ACTB77) and Amycolatopsis sp. (ACTB-290) against C. musae.

\section{SEM imaging of C. musae hyphae}

The contents of the Petri dishes from the fifth day of the aforementioned assays (control and co-culture) were used to investigate the mVOCs effects on the morphological structure of the fungus hyphae by scanning electron microscopy (SEM), Figure 4.

Figure 4a shows the perfect stage of $C$. musae hyphae under axenic cultured (control), with fungal cell structure having straight, cylindrical and long hyaline (non-septate hyphae), with their walls remaining smooth and shiny. ${ }^{44}$ The effect of VOCs from actinobacteria ACTB-77 strain on $C$. musae hyphae is observed in Figure 4b. Although most of the hyphae showed wrinkled and withered aspects, a small portion of cell filaments was partially unharmed, and no breakage of the hyphae was observed. SEM image of fungus hyphae from co-culture experiment with ACTB-290 strain is depicted in Figure 4c. In this case, the deformation of all filaments of residual cells, with wrinkled, withered (loss of turgor) and brittle aspects were observed, suggesting a possible leakage of the intracellular material.

Thus, SEM imaging analysis of $C$. musae hyphae revealed that VOCs produced by the actinobacteria strains 

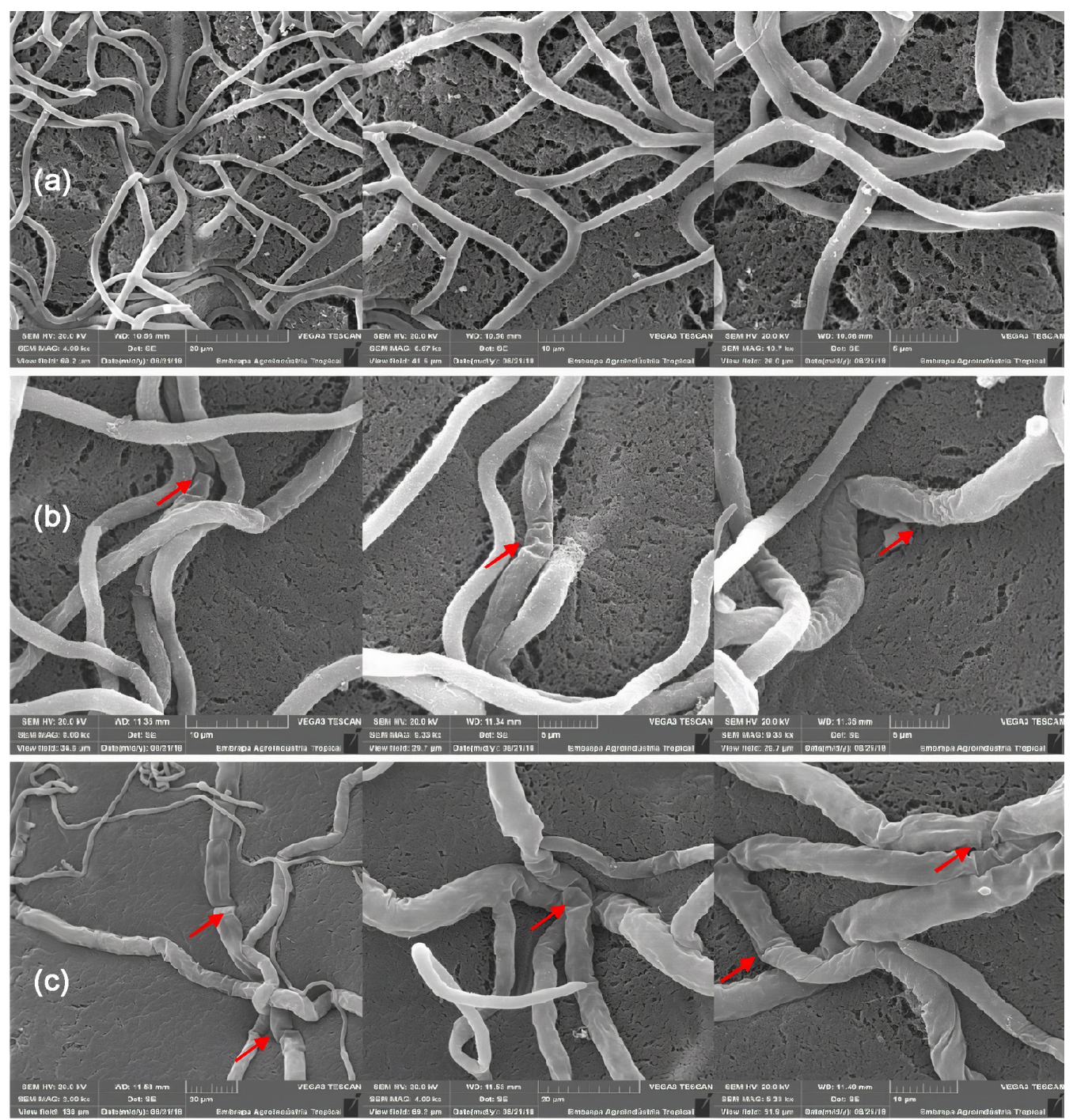

Figure 4. SEM imaging $(20 \mu \mathrm{M})$ of $C$. musae hyphae in the fifth day of experiment: (a) under axenic culture (control); (b) in co-culture with Streptomyces sp.; (c) in co-culture with Amycolatopsis sp.

during co-culture experiments promoted deformation of the fungal cell filaments. The highest antifungal activity of VOCs from Amycolatopsis sp. (ACTB-290) was corroborated through the greatest damage observed in the fungal hyphae image.

Antifungal activity of VOCs from Amycolatopsis sp. (ACTB-290) against Lasiodiplodia and Colletotrichum strains

The antifungal potential of VOCs from Amycolatopsis sp. against $C$. musae motivated the investigation of the activity of this strain against other species of phytopathogenic fungi, one from Colletotrichum genus (C. brevisporum) and five species of Lasiodiplodia (L. brasiliense, L. theobramae, L. harmozganensis, L. brasiliense and L. viticola), Figure 5.
Among the tested fungal strains, the VOCs of ACTB-290 presented significant growing inhibition (GI $>50 \%$ ) of C. brevisporum (GI 76.0\%), L. theobramae (GI 68.0\%) and L. harmozganensis (GI 54.4\%). Comparison of these results with those obtained previously against $C$. musae (GI $80.5 \%$ ) suggests that the actinobacteria is more selective towards Colletotrichum species.

Identification of VOCs produced by Amycolatopsis sp. (ACTB-290) and Streptomyces sp. (ACTB-77) under axenic culture and co-culture with C. musae

Optimization of the VOCs extraction from Amycolatopsis sp. (ACTB-290) by HS-SPME

As already mentioned, studies on $\mathrm{mVOCs}$ first required experimental optimization by varying fiber coating and headspace conditions. ${ }^{23}$ Thus, experimental optimization 

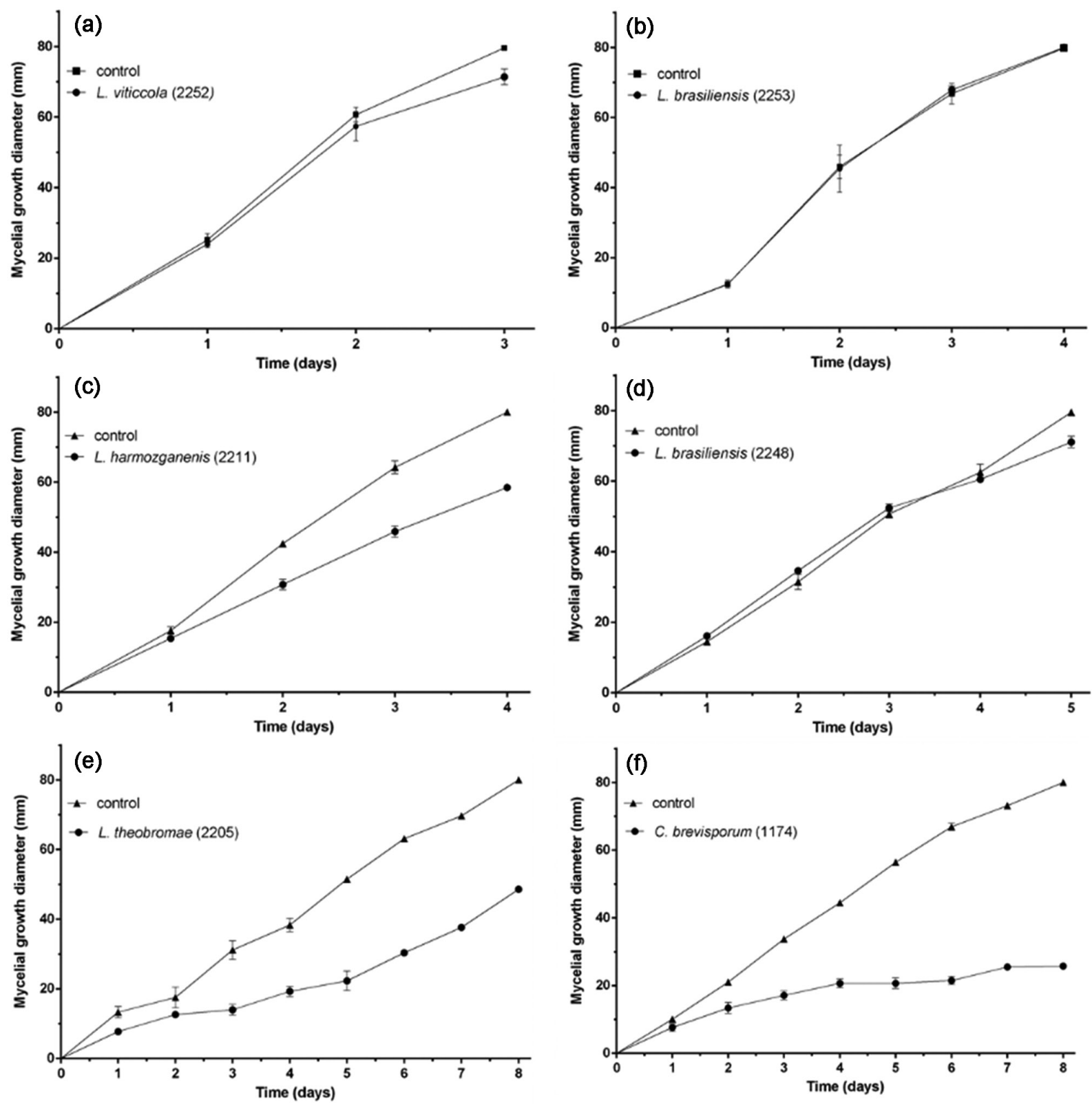

Figure 5. Results from the antifungal activity of Amycolatopsis sp. (ACTB-290) against: (a) Lasiodiplodia viticola; (b) L. brasiliensis-2253; (c) L. brasiliensis-2248; (d) L. theobromae; (e) Colletotrichum brevisporum. Fungus strain under axenic culture is the control experiment.

related to the actinobacteria ACTB-290 involved varying fiber coating (PDMS/DVB/CAR, PDMS/DVB and PDMS/CAR), the extraction time (10, 20, 30 and $40 \mathrm{~min})$ and temperature $\left(30\right.$ and $\left.50{ }^{\circ} \mathrm{C}\right)$. Microbial VOCs were analyzed by GC-MS and evaluated in relation to the quantity and area of the peaks in each experiment. Based on the variations of the observed error, it was concluded that temperature is a significant factor, being more important than extraction time. Analysis of the interactions between fiber, extraction time and temperature, however, revealed that, for this matrix, these variables are independent. After all data analyses of the optimization experiments of the mVOC extraction of ACTB-290 by HS-SPME, the optimum conditions were established as: fiber coating $\mathrm{PDMS} / \mathrm{DVB} / \mathrm{CAR}$, extraction time $30 \mathrm{~min}$ and extraction temperature $50{ }^{\circ} \mathrm{C}$.

\section{Identification of VOCs produced by the microorganisms}

The actinobacteria strains, Streptomyces sp. (ACTB-77) and Amycolatopsis sp. (ACTB-290), and the phytopathogen fungus $C$. musae were cultured (axenic and co-culture conditions) in PDA medium for mVOCs production. For all microorganisms, HS-SPME experiments were performed using the optimized conditions (PDMS/DVB/CAR, $50{ }^{\circ} \mathrm{C}$ and $30 \mathrm{~min}$ ). Analyses of their respective VOCs were done by GC-MS and their compositions are displayed at Table 2 . 
Table 2. Volatile organic compounds (VOCs) produced by the rhizosphere actinobacteria Streptomyces sp. (ACTB-77) and Amycolatopsis sp. (ACTB-290) strains, and the phytopathogen fungus Colletotrichum musae (CM) under axenic and co-culture conditions. Compounds listed and numbered (column No.) by crescent order of their RI

\begin{tabular}{|c|c|c|c|c|c|c|c|}
\hline \multicolumn{3}{|c|}{ Compound } & \multicolumn{3}{|c|}{ Axenic culture } & \multicolumn{2}{|c|}{$\frac{\text { Co-culture }}{\text { Peak area } \pm \text { SD } / \%}$} \\
\hline No. & Name & $\mathrm{RI}\left(\mathrm{RI}^{\mathrm{a}}\right)$ & $\mathrm{CM}$ & ACTB-77 & ACTB-290 & $\mathrm{CM}+\mathrm{ACTB}-77$ & CM + ACTB-290 \\
\hline 1 & 3-methyl-butan-1-ol & $731(731)$ & $23.82 \pm 2.07$ & $1.77 \pm 0.11$ & - & $6.42 \pm 0.75$ & $5.76 \pm 0.09$ \\
\hline 2 & 2-methyl-butan-1-ol & $732(732)$ & $5.85 \pm 0.31$ & $0.98 \pm 0.08$ & - & $2.43 \pm 0.26$ & $2.51 \pm 0.02$ \\
\hline 3 & 3-methyl-pentan-2-one & 747 (750) & - & $2.03 \pm 0.04$ & - & - & - \\
\hline 4 & dimethyl disulfide & $752(751)$ & - & - & $48.49 \pm 3.48$ & - & $2.18 \pm 0.68$ \\
\hline 5 & 3-methylbutan-1-ol acetate & $869(869)$ & $0.96 \pm 0.04$ & - & - & - & - \\
\hline 6 & 2,4-dithiapentane & $893(892)$ & & & $4.32 \pm 0.85$ & & $0.58 \pm 0.11$ \\
\hline 7 & 6-methyl-heptan-2-one & 947 (949) & - & - & $2.47 \pm 0.07$ & - & $0.77 \pm 0.06$ \\
\hline 8 & dimethyl trisulfide & $976(976)$ & & & $21.14 \pm 3.50$ & & $0.76 \pm 0.25$ \\
\hline 9 & $\beta$-myrcene & $980(988)$ & $1.14 \pm 0.22$ & $4.95 \pm 0.65$ & & $1.33 \pm 0.02$ & $0.66 \pm 0.07$ \\
\hline 10 & not identified & 985 & & & $0.94 \pm 0.04$ & & $0.36 \pm 0.05$ \\
\hline 11 & 2-methylenebornane & 990 (n.f.) $^{\mathrm{b}}$ & & & $1.13 \pm 0.04$ & & $0.78 \pm 0.20$ \\
\hline 12 & $\alpha$-phellandrene & 994 (1002) & $8.09 \pm 1.72$ & - & - & $11.94 \pm 0.39$ & $11.91 \pm 0.65$ \\
\hline 13 & $\alpha$-terpinene & $1008(1014)$ & $3.43 \pm 0.74$ & - & - & $4.22 \pm 0.05$ & $6.02 \pm 0.21$ \\
\hline 14 & p-cymene & $1017(1020)$ & $3.16 \pm 0.47$ & - & - & $3.50 \pm 0.11$ & $3.47 \pm 0.28$ \\
\hline 15 & $\beta$-phellandrene & $1023(1025)$ & $28.38 \pm 4.89$ & - & - & $43.01 \pm 1.53$ & $44.71 \pm 2.61$ \\
\hline 16 & 2-methyl-2-bornene & $1023(1021)$ & & & $2.42 \pm 0.26$ & & \\
\hline 17 & trans- $\beta$-ocimene & $1042(1044)$ & & $2.41 \pm 0.35$ & - & - & - \\
\hline 18 & methyl 2-ethylhexanoate & $1048(1043)$ & & & $2.49 \pm 0.04$ & & $0.53 \pm 0.01$ \\
\hline 19 & not identified & 1058 & & & $2.13 \pm 0.74$ & & $1.29 \pm 0.54$ \\
\hline 20 & 1-octanol & $1064(1063)$ & & $1.52 \pm 0.24$ & & & \\
\hline 21 & linalool & $1092(1095)$ & & $38.53 \pm 5.19$ & & $6.08 \pm 0.49$ & \\
\hline 22 & 2-nonanone & $1094(1090)$ & & & $1.14 \pm 0.24$ & & $2.40 \pm 0.10$ \\
\hline 23 & 2-nonanol & $1102(1097)$ & & & & & $0.47 \pm 0.04$ \\
\hline 24 & phenylethyl alcohol & $1106(1107)$ & $7.44 \pm 0.62$ & & & $1.97 \pm 0.32$ & $3.31 \pm 0.30$ \\
\hline 25 & allo-ocimene & $1123(1127)$ & & $0.71 \pm 0.11$ & & & \\
\hline 26 & not identified & 1129 & & $1.7 \pm 0.23$ & & & \\
\hline 27 & $\begin{array}{l}\text { methyl(methylthio)methyl } \\
\text { disulfide }\end{array}$ & 1135 (1134) & & & $1.95 \pm 0.07$ & & \\
\hline 28 & $p$-menthone & $1148(1148)$ & $0.97 \pm 0.26$ & & & $1.26 \pm 0.10$ & $1.93 \pm 0.06$ \\
\hline 29 & $p$-mentha-1,5-dien-8-ol & $1160(1165)$ & $0.72 \pm 0.06$ & & & $0.43 \pm 0.08$ & \\
\hline 30 & 2-decanone & $1162(1167)$ & & & $0.80 \pm 0.07$ & & $0.92 \pm 0.12$ \\
\hline 31 & menthol & $1166(1167)$ & $1.39 \pm 0.15$ & & & $0.31 \pm 0.03$ & $0.58 \pm 0.11$ \\
\hline 32 & 2-methylisoborneol & $1177(1180)$ & & & $2.27 \pm 0.05$ & & $1.51 \pm 0.24$ \\
\hline 33 & not identified & 1182 & & & $0.76 \pm 0.09$ & & \\
\hline 34 & $p$-mentha-1(7),2-dien-8-ol & $1184(1186)$ & $1.64 \pm 0.15$ & & & & $0.62 \pm 0.15$ \\
\hline 35 & $\alpha$-terpineol & $1186(1186)$ & & $1.10 \pm 0.63$ & & $1.68 \pm 0.08$ & \\
\hline 36 & thymol methyl ether & $1228(1230)$ & & $5.06 \pm 0.55$ & & $0.95 \pm 0.05$ & \\
\hline 37 & carvacrol methyl ether & $1235(1240)$ & & $4.24 \pm 0.68$ & & $0.64 \pm 0.03$ & \\
\hline 38 & geraniol & 1249 (1249) & & $1.86 \pm 0.43$ & & $0.49 \pm 0.03$ & \\
\hline 39 & not identified & 1254 & & $1.55 \pm 0.25$ & & $0.80 \pm 0.09$ & \\
\hline 40 & not identified & 1261 & & & $1.64 \pm 0.17$ & & $0.42 \pm 0.09$ \\
\hline 41 & 1-decanol & $1264(1266)$ & & $0.79 \pm 0.07$ & & & \\
\hline 42 & not identified & 1284 & & $0.75 \pm 0.04$ & & $0.23 \pm 0.01$ & \\
\hline 43 & 2-undecanone & $1296(1293)$ & & & $0.93 \pm 0.11$ & & $0.35 \pm 0.05$ \\
\hline 44 & 2-undecanol & 1303 (1301) & & & & & $0.65 \pm 0.05$ \\
\hline 45 & not identified & 1330 & & $0.52 \pm 0.05$ & & & \\
\hline 46 & silphinene & 1335 (1339) & $0.42 \pm 0.06$ & & & $1.33 \pm 0.49$ & $0.43 \pm 0.02$ \\
\hline 47 & not identified & 1337 & & $0.71 \pm 0.10$ & & & \\
\hline 48 & 2-dodecanone & $1362(1361)$ & & & $1.45 \pm 0.05$ & & $0.19 \pm 0.05$ \\
\hline 49 & not identified & 1370 & & & $0.87 \pm 0.04$ & & $0.48 \pm 0.05$ \\
\hline 50 & not identified & 1375 & & & & & $0.28 \pm 0.03$ \\
\hline 51 & not identified & 1401 & & $1.69 \pm 1.19$ & & $0.66 \pm 0.05$ & \\
\hline
\end{tabular}


Table 2. Volatile organic compounds (VOCs) produced by the rhizosphere actinobacteria Streptomyces sp. (ACTB-77) and Amycolatopsis sp. (ACTB-290) strains, and the phytopathogen fungus Colletotrichum musae (CM) under axenic and co-culture conditions. Compounds listed and numbered (column No.) by crescent order of their RI (cont.)

\begin{tabular}{|c|c|c|c|c|c|c|c|}
\hline \multirow{2}{*}{\multicolumn{3}{|c|}{ Compound }} & \multirow{2}{*}{\multicolumn{3}{|c|}{$\begin{array}{c}\text { Axenic culture } \\
\text { Peak area } \pm \text { SD } / \%\end{array}$}} & \multirow{2}{*}{\multicolumn{2}{|c|}{$\begin{array}{c}\text { Co-culture } \\
\text { Peak area } \pm \text { SD } / \%\end{array}$}} \\
\hline & & & & & & & \\
\hline No. & Name & $\mathrm{RI}\left(\mathrm{RI}^{\mathrm{a}}\right)$ & $\mathrm{CM}$ & ACTB-77 & ACTB-290 & $\mathrm{CM}+\mathrm{ACTB}-77$ & CM + ACTB-290 \\
\hline$\overline{52}$ & geosmin & $1410(1399)$ & & $17.25 \pm 2.55$ & & $3.08 \pm 0.16$ & \\
\hline 53 & not identified & 1446 & & & & & $0.30 \pm 0.04$ \\
\hline 54 & 2-tridecanone & $1464(1467)$ & & & $0.67 \pm 0.04$ & & $0.31 \pm 0.07$ \\
\hline 55 & not identified & 1470 & & $1.03 \pm 0.14$ & & & \\
\hline 56 & not identified & 1470 & & & & & $0.39 \pm 0.03$ \\
\hline 57 & $\beta$-chamigrene & $1476(1476)$ & $0.91 \pm 0.19$ & & & $0.48 \pm 0.05$ & $0.39 \pm 0.05$ \\
\hline 58 & $\gamma$-gurjunene & 1479 (1479) & & $0.39 \pm 0.08$ & & & \\
\hline 59 & aristolochene & $1486(1487)$ & $7.56 \pm 1.41$ & & & $3.50 \pm 0.19$ & \\
\hline 60 & $\alpha$-selinene & $1498(1498)$ & $3.60 \pm 0.75$ & & & $1.76 \pm 0.21$ & $1.54 \pm 0.20$ \\
\hline 61 & viridiflorene & $1501(1497)$ & & $0.39 \pm 0.06$ & & & \\
\hline 62 & not identified & 1515 & & $0.73 \pm 0.13$ & & & \\
\hline 63 & 7-epi- $\alpha$-selinene & $1522(1520)$ & $0.53 \pm 0.11$ & & & $0.47 \pm 0.05$ & $0.20 \pm 0.02$ \\
\hline 64 & selina-3,7(11)-diene & $1539(1542)$ & & $6.92 \pm 0.09$ & & $1.43 \pm 0.12$ & \\
\hline 65 & 2-tetradecanone & $1565(1570)$ & & & $1.02 \pm 0.03$ & & \\
\hline 66 & (Z)-8-dodecen-1-ol acetate & $1588(1588)$ & & & $0.13 \pm 0.02$ & & \\
\hline 67 & 1-tetradecanol & $1643(1647)$ & & & $0.83 \pm 0.07$ & & \\
\hline 68 & $\alpha$-eudesmol & $1647(1652)$ & & $0.26 \pm 0.02$ & & & \\
\hline 69 & mint sulfide & $1738(1740)$ & & $0.20 \pm 0.02$ & & & \\
\hline
\end{tabular}

RI: retention index from literature; 38 balthough the RI from literature is missing for compound 11, it was identified by comparison of its mass spectra with that published in the literature ${ }^{38}$ for the compound 2-methylenebornane. RI: retention index; n.f.: not found; SD: standard deviation.

Mass spectra of the microbial produced compounds are available in Figure S5, SI section.

\section{mVOCs profile under axenic culture}

\section{Fungus C. musae}

The study on VOCs produced by $C$. musae under axenic culture resulted in 18 recorded peaks, all of them identified by GC-MS analysis (Table 2). Among them, 3-methyl-butan-1-ol (1) and $\beta$-phellandrene (15) were found as the main compounds. These two compounds, together with $\alpha$-phellandrene (12), phenylethyl alcohol (24) and aristolochene (59) represent ca. $75 \%$ of the mVOCs peak area. Constituents from C. musae were distributed into three different cases, non-terpenoids (4 compounds; ca. $38 \%$ total area), monoterpenes (9 compounds; ca. $49 \%$ total area); sesquiterpenes (5 compounds; ca. $13 \%$ total area). No reports on VOCs produced by either C. musae or any other congener species were found in the literature for comparison purposes. Instead, the literature reports studies on VOCs either from bacteria strains with inhibition of Colletotrichum species. ${ }^{45,46}$ or from plants/ fruits contaminated with some fungus strains. ${ }^{47,48}$

\section{Actinobacteria Streptomyces sp. (ACTB-77)}

The study on VOCs produced by ACTB-77 strain under axenic culture yielded 27 peaks recorded in the
GC chromatogram (Table 2). Among them, $70 \%$ of the compounds (19 peaks) were identified, together representing ca. $91 \%$ of the total area of all recorded peaks. The monoterpene linalool (21) and the sesquiterpene geosmin (52) were found as the main constituents, these two compounds accounting for ca. $56 \%$ of the mVOCs peak area. The identified compounds included non-terpenoids (6 compounds; ca. $8 \%$ total area), monoterpene ( 9 compounds; ca. $76 \%$ total area) and sesquiterpene (5 compounds; ca. $8 \%$ total area). Sesquiterpene mint sulfide (69) was the only sulfur-containing compound produced by ACTB-77.

Some of these non-terpenoid compounds have been reported as by-products from primary metabolic pathways of actinomycetes. ${ }^{8}$ Previous investigations of VOCs profiling of Streptomyces isolates have been reported in literature. ${ }^{17,49}$ A total of twenty-six Streptomyces strains were assayed for their ability to produce VOCs. ${ }^{17}$ In that study, 53 compounds were identified out of the 120 detected peaks. The compounds were classified as alkanes, alkenes, alcohols, esters, ketones, sulfur-compounds, and terpenes. As for ACTB-77, 3-methyl-butan-1-ol (1), 2-methyl-1-butanol (2), and geosmin (52) were identified as among the most frequent compounds produced by the microorganisms. A similar study involved VOCs production of twelve Streptomyces strains, eleven of them from rhizosphere. Besides the presence of alcohols, aldehydes 
and terpenes, geosmin (52) was the common constituent in all isolates. ${ }^{49}$

The production of the monoterpene linalool (21) as major constituent by ACTB-77 commanded special attention in our work since a similar result was found only in previous studies on VOCs produced by two strains of S. philanthi, RM$1-138^{19}$ and RL-1-178, ${ }^{50}$ both isolated from the rhizosphere soil of chili peppers in Thailand. Among the 39 mVOCs produced by RL-1-178 strain under axenic cultured in wheat seed medium, linalool $(\mathbf{2 1} ; \mathbf{1 3 . 5 5 \% )}$ ) and geosmin $(\mathbf{5 2} ; 13.75 \%)$ were also found as the major compounds. ${ }^{50}$ Linalool $(21 ; 9.06 \%)$ was the main compound produced by RM-1-138 strain under the same experimental conditions, while geosmin (52) was found in only $1.23 \%$.

S. clavuligerus linalool synthase (bLinS) was discovered and identified as a catalyst for linalool production using a metabolic engineering platform. ${ }^{51}$ The use of bLinS in metabolically engineered monoterpene-producing $E$. coli strains yielded a 300-fold higher linalool production compared with the corresponding linalool synthase from plants. Therefore, the identification of this monoterpene as major constituent produced by ACTB-77 and S. philanthi (RM-1-138 and RL-1-178 strains), ${ }^{19,50}$ may represent examples of linalool synthase expression in Streptomyces strains. It is worth highlighting that linalool has high commercial value since it is used in fragrances, cosmetic and non-cosmetic products, as well as an intermediate in organic syntheses; the use of commercial linalool exceeds more than 1,000 metric tons per year worldwide. ${ }^{51}$

Comparison between VOCs produced by the actinobacteria ACTB-77 and those identified for the fungus C. musae, revealed compounds 3-methyl-butan-1-ol (1), 2-methyl-butan-1-ol (2) and $\beta$-myrcene (9) as the only chemical constituents common to both the microorganisms. Nevertheless, although alcohol 1 was a major compound produced by the fungus $(23.82 \pm 2.07 \%)$, it was found at only $1.77 \pm 0.11 \%$ in the actinobacteria VOCs peak area. Both microorganisms produced $\beta$-myrcene (9) in low concentrations, $1.14 \pm 0.22 \%$ (C. musae) and $4.95 \pm 0.65 \%$ (ACTB-77 strain).

\section{Actinobacteria Amycolatopsis sp. (ACTB-290)}

The study on VOCs produced by ACTB-290 strain under axenic culture provided 22 peaks recorded in the GC chromatogram (Table 2). From these, 17 peaks $(77 \%$ of the peaks) were identified, accounting for ca. $94 \%$ of the total area of all recorded peaks. Dimethyl disulfide (4) and dimethyl trisulfide (8) were the major compounds, together representing ca. $70 \%$ of the VOCs peak area. Besides these two, 2,4-dithiapentane (6) and methyl(methylthio)methyl disulfide (27) were also identified as sulfur-containing compounds. The identified compounds included four sulfur-derivatives (ca. 76\% total area), nine non-terpenoids (ca. 12\% total area) and three bicyclic monoterpenes (ca. 6\% total area).

Actinobacteria from Streptomyces and Amycolatopsis genera are known to be proficuous sources of non-volatile antibiotic compounds. ${ }^{52}$ Nevertheless, different from Streptomyces that has a VOCs profile reported from some of its species, no study on VOCs identification of Amycolatopsis species was found in the literature. A significant difference was clearly observed between the VOCs profile of these two actinobacteria strains. While Streptomyces sp. (ACTB-77) produced terpenes (21 and 52) as main constituents, Amycolatopsis sp. (ACTB-290) yielded sulfur-containing compounds (4 and 8) as predominant in its VOCs peak area. It should be said that, although dimethyl disulfide (4) and dimethyl trisulfide (8) were not produced by Streptomyces sp. (ACTB-77), they have been included as two common constituents on the list of putative VOCs from eleven Streptomyces strains isolated from a Rhizoctonia-suppressive soil, as well as from a strain of $S$. lividans. ${ }^{49}$ Additionally, comparison between VOCs profiles of actinobacteria ACTB-290 and C. musae showed no common compounds for these two microorganisms.

\section{mVOCs profile under co-culture}

\section{Co-culture of ACTB-77 and C. musae}

During the co-culture experiment for mVOCs extraction by HS-SPME, inhibition of the growing fungus could be observed, corroborating the already discussed antifungal activity of ACTB-77 against C. musae. GC-MS analysis of the VOCs produced by the microorganisms in the co-culture experiment yielded 26 peaks (Table 2). Among them, only 3 compounds (ca. $1.7 \%$ of the total area) were not identified.

As already mentioned, compounds 3-methyl-butan1-ol (1), 2-methyl-butan-1-ol (2) and $\beta$-myrcene (9) were the only VOCs produced by both microorganisms under axenic culture. These three compounds were also identified in the co-culture. Excluding these compounds, comparison of the VOCs profile of the actinobacteria under axenic culture ( 24 exclusive peaks) and that from the co-culture with the fungus revealed only ten compounds from ACTB-77. These were: linalool (21), $\alpha$-terpineol (35), thymol methyl ether (36), carvacrol methyl ether (37), geraniol (38), geosmin (52) and selina-3,7(11)-diene (64), besides three non-identified. Therefore, fourteen VOCs produced by the actinobacteria under axenic culture were not detected in the co-culture 
experiment. It is worth highlighting that, $\mathbf{2 1}$ and $\mathbf{5 2}$, both major compounds produced by the actinobacteria under axenic culture, showed a lower percentage of peak area in the co-culture.

$\alpha$-Phellandrene (12) and $\beta$-phellandrene (15), both compounds identified as $C$. musae VOCs under the axenic culture, were the major constituents in the co-culture. In this latter experiment, a considerable increment in the production of $\mathbf{1 5}$ by the fungus was observed. Studies from the literature revealed the antibacterial activity of fruit and plant essential oils with a high content of the sesquiterpene $15 .{ }^{53,54}$ This suggests that the increment of this compound in the co-culture may be a fungal defense.

The antifungal activity of VOCs produced by Streptomyces sp. (ACTB-77) may be due to the presence of monoterpenes (especially linalool, 21) with its bioactivity already reported in the literature. ${ }^{55-59}$

\section{Co-culture of ACTB-290 and C. musae}

As observed for the co-culture experiment of ACTB-77 and C. musae, during the VOCs extraction of ACTB-290 co-cultured with the same fungus inhibition of the fungus growing. GC-MS analysis of the VOCs produced in the co-culture experiment recorded 36 peaks, with 29 of them (81\%; ca. $96 \%$ of the total area) being identified (Table 2). Comparison of the peaks recorded in this experiment with those from the axenic cultures of the microorganisms showed that 16 peaks (4 non-identified compounds) were related to compounds produced by the actinobacteria, 15 peaks were produced by the fungus, and 5 peaks (3 non-identified compounds) were related exclusively to the co-culture.

Among the 22 VOCs produced by the actinobacteria ACTB-290 cultured under axenic condition, only 2-methyl-2-bornene (16), methyl(methylthio)methyl disulfide (27), 2-tetradecanone (65), (Z)-8-dodecen-1-ol acetate (66) and 1-tetradecanol (67), besides one of the nonidentified compounds $\left(t_{R} 10.87 \mathrm{~min}\right)$, were not observed in the co-culture. Concerning C. musae, only compounds 3-methylbutan-1-ol acetate (5), $p$-menta-1,5-dien-8-ol (29) and aristolochene (59), which were produced by the fungus under axenic culture, were not detected in the co-culture. Again, the fungal VOCs $\alpha$-phellandrene (12) and $\beta$-phellandrene (15) were the major constituents in the co-culture. There was a significant increase in peak area for the fungal compound $\alpha$-terpinene (13) in the co-culture, compared with the axenic culture.

Differently from what had been observed in the coculture of the fungus and the actinobacteria ACTB-77, the study involving strain ACTB-290 yielded five exclusive peaks, that include the two identified mVOCs 2-nonanol (23) and 2-undecanol (44). Interestingly, these alcohols may be thought as bioreduction products of the respective $\mathrm{C}_{9}$ and $\mathrm{C}_{11}$ ketones (VOCs from actinobacteria) from the fungus. Thus, the antifungal activity of Amycolatopsis sp. (ACTB-290) against $C$. musae may be associated to its sulfur-containing metabolites dimethyl disulfide (4) and dimethyl trisulfide $(\mathbf{8})$, both previously reported as potent fungicides. ${ }^{60,61}$

\section{Statistical analysis of the experiments}

The statistical analysis discussed in this study was confined to a comparison between the relative concentrations of the peak areas of each compound produced by the microorganisms under axenic culture and those produced in the co-culture (ACTB-77 and C. musae; ACTB-290 and C. musae). A principal component analysis (PCA) ${ }^{62}$ was performed, producing an unsupervised pattern recognition algorithm used to represent a high number of results through two graphics called scores and loadings. In PCA, the scores graph presents the similarity/differences between each culture while the loadings graphic displays the constituent that is important for differences and similarities between each group. In summary, all peaks are converted into new variables, called PCs, each one accounting for the data variability. Thus, similar samples are close in the scores graphic while different samples are distant. The relative concentration of each constituent is displayed in loadings graphics, which have the same axes as in the scores graphics. When the scores and loadings graphics are overlaid, it is possible to deduce that the relative concentration of a constituent will be higher for the sample with high scores. Each number displayed in loadings graphics represents a peak which it is identified in Table 2, and which was listed in increasing order of the retention index.

\section{ACTB-77, C. musae and their co-culture}

PC1 and PC2 explain $80.36 \%$ of the data variance (Figure 6), making it possible to analyze all constituents in the samples using only these two variables. A natural separation tendency was observed among the groups, which are represented by yellow (ACTB-77 strain), dark blue (C. musae strain) and light blue (co-culture) colors (Figure 6a). In this case, the highest separation was observed for the samples from the axenic culture of the actinobacteria since these samples presented the highest scores values in PC1 (Figure 6a). The relative concentrations of the constituents are represented in the loadings graph (Figure 6b) by circles with three different colors, where yellow, dark blue and light blue are associated to the highest concentration 

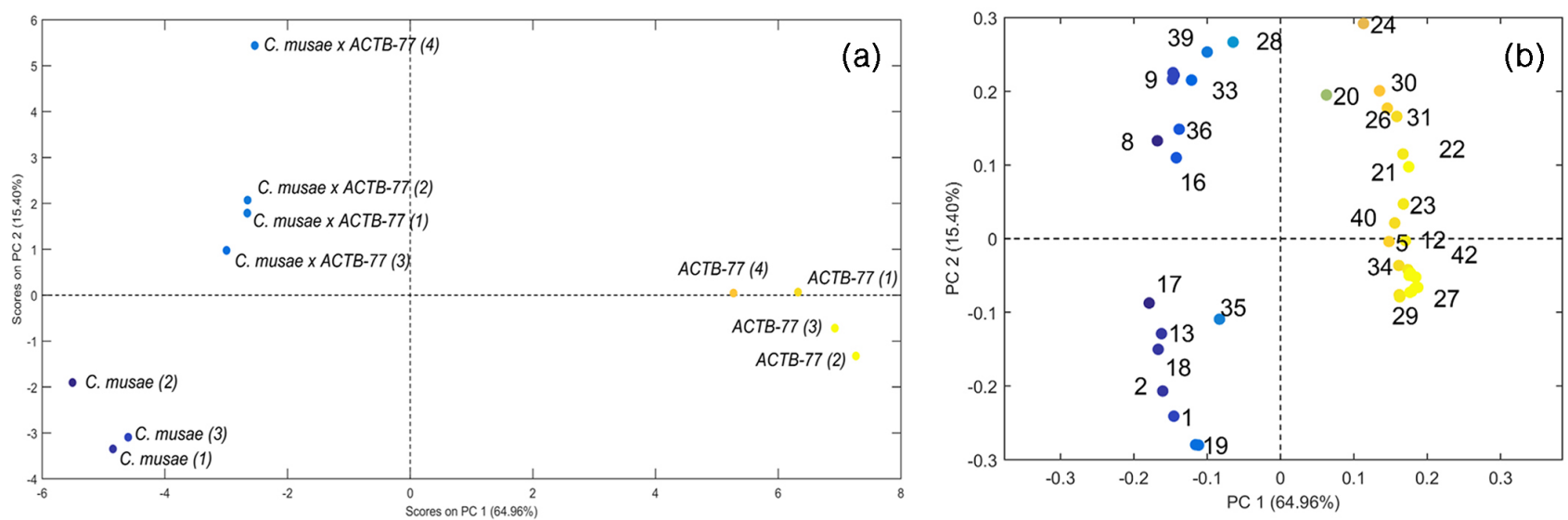

Figure 6. Scores (a) and loadings (b) graphs obtained by PC1 and PC2 for the samples of ACTB-77 (yellow), C. musae (dark blue) and the co-culture (light blue).

constituents from the actinobacteria (ACTB-77), C. musae and co-culture, respectively.

Figure $6 \mathrm{~b}$ shows 3-methyl-butan-1-ol (1), 2-methylbutan-1-ol (2), $\alpha$-phellandrene (12), $\beta$-phellandrene (15), phenylethyl alcohol (24), p-mentha-1,5-dien8-ol (29), menthol (31) and aristolochene (59) as the constituents of the $C$. musae group with the highest relative concentrations. Among them, compounds 1, 12, 15, 24 and 59 represent ca. $75 \%$ of the VOCs peak area of the fungus. Concerning the constituents from the ACTB-77 group, geraniol (38), 1-decanol (41), non-identified (47), geosmin (52), $\gamma$-gurjunene (58) and mint sulfide (69) are displayed in Figure $6 \mathrm{~b}$ as those mVOCs with the highest relative concentrations. Although $\mathbf{3 8}$ and $\mathbf{5 2}$ are found in both co-culture and ACTB-77 axenic culture, these constituents had higher concentrations in the latter experiment. The $\alpha$-phellandrene (12), $\beta$-phellandrene (15) and silphinene (46), already found in axenic culture of C. musae, had their relative concentrations intensified in the co-culture experiment (Figure 6b).

$\mathrm{PC} 1$ scores were not significant enough to differentiate between samples of $C$. musae and co-culture. It is possible to observe a correlation between the variables highlighted in blue (dark and light) in the loadings graph (Figure 6b). The 3-methyl-butan-1-ol (1) and the $\beta$-phellandrene (15), both found as main VOCs produced by $C$. musae, are the most important constituents for distinguishing the fungus from the co-culture. These two chemical constituents are not correlated, and they present significant difference in their relative concentrations in the two groups (C. musae and co-culture). The differentiation between $C$. musae and co-culture groups can be observed through the different values of their PC2 scores (Figure 6a).

In order to confirm the differences between the groups, a $t$-test was performed using the scores of PC1 and PC2. In this case, it was observed that there were statistically significant differences between C. musae, ACTB-77 and their co-culture.

\section{ACTB-290, C. musae and their co-culture}

Together, PC1 and PC2 explain $76.84 \%$ of the data variance (Figure 7), enabling an analysis of similarities/ differences of the groups using only these two variables. Comparison of this study with those previously discussed for the experiments involving actinobacteria ACTB-77 revealed a higher separation tendency of the groups for the studies with ACTB-290, that are represented by dark blue (ACTB-290 strain), yellow (C. musae strain) and three different colors (orange, light green and green) for co-culture (Figure 7a). The use of three colors for co-culture experiments were needed to indicate different composition in their replicates.

As observed in the loadings graph (Figure 7b), the lowest scores of PC1 are the variables highlighted in dark blue, which are related to the axenic culture of ACTB-290. In this graph, dimethyl disulfide (4), dimethyl trisulfide (8) and methyl 2-ethylhexanoate (18), Table 2, are significant variables for distinguishing ACTB-290 from the other two groups (C. musae and co-culture). Additionally, these three chemical constituents are found in relatively higher concentrations in the actinobacteria axenic culture when compared with the co-culture. $\alpha$-Phellandrene (12), $\alpha$-terpinene (13) and $\beta$-phellandrene (15), in Table 2, which are represented in the loadings graph (Figure 7b) with orange (compounds 12 and 15) and green (compound 13) circles, presenting positive values of scores in PC2. This agrees with the fact that these mVOCs had a significant increase in the co-culture experiment when compared to the axenic culture of the fungus.

The sulfur-containing compounds dimethyl disulfide (4), dimethyl trisulfide (8) and methyl(methylthio)methyl disulfide (27), in Table 2, all VOCs exclusively produced 

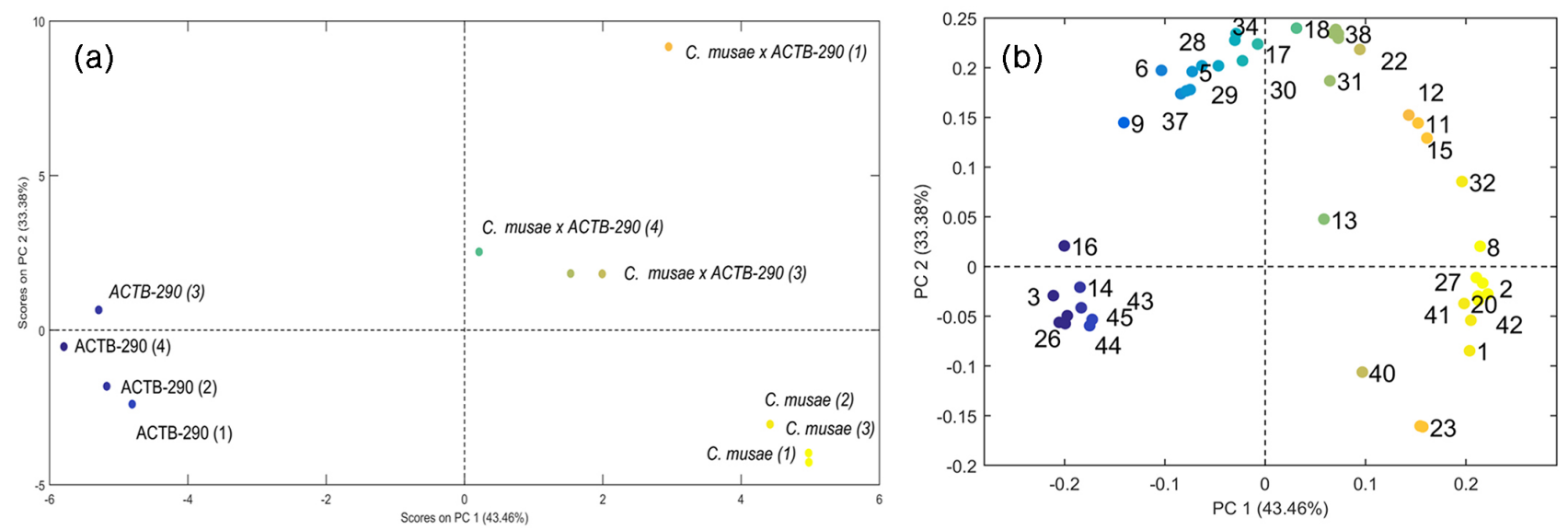

Figure 7. Scores (a) and loadings (b) graphs obtained by PC1 and PC2 for the samples of ACTB-290 (dark blue), C. musae (yellow) and the co-culture (orange, light green and green).

by the actinobacteria ACTB-290, are reported as potent fungicides. Interestingly, no correlation between these compounds and the alcohols ( 23 and $\mathbf{4 4}$, in Table 2) recorded exclusively in the co-culture experiment was observed in the correlation map (Figure S6, SI section). This suggest that these alcohols are products of bioreduction of the actinobacterial produced ketones 22 and $\mathbf{4 3}$, respectively, by the fungus strain.

A $t$-test was also performed with the scores and revealed that ACTB-290 strain is statistically different from the fungal strain. This latter microorganism presented a significant difference when compared to the co-culture (C. musae/ACTB-290) in the PC2 score, but not in PC1 $\left(t=1.6696 / t_{\text {crit }}=2.447\right.$ and $\left.p=0.1460\right)$. This corroborates the result already discussed, which showed that the co-culture (C. musae/ACTB-290) is very similar to the axenic culture of ACTB-290, especially for the compounds highlighted in PC1.

\section{Global analysis}

A global analysis of the aforementioned experiments was performed since, different from a non-statistical approach, PCA enables evaluation of all samples (ACTB-77, ACTB-290, fungus and co-cultures) grouped in the same graphs (Figure 8). In this case, PC1, PC2 and $\mathrm{PC} 3$ explain $75.83 \%$ of the data variance, allowing the analysis of similarities/differences of the groups using these three variables. A strong separation tendency of the groups was observed, represented by yellow (ACTB-77), blue (ACTB-290), cyan (C. musae) and light green (ACTB-77/C. musae) and dark blue and blue (ACTB-290/C. musae) colors in the scores graphs depicted in Figure 8a (PC1 vs. PC2) and Figure 8b (PC1 vs. PC3). The ellipses are arbitrarily included to indicate the differences between the classes. The statistical differences were calculated using the $t$ and $F$ hypotheses tests.
As observed in Figures $8 \mathrm{a}$ and $8 \mathrm{~b}$, co-culture ACTB-77/C. musae is more similar to the fungus group than to the actinobacteria group. Concerning the co-culture involving the actinobacteria ACTB-290 (ACTB-290/C. musae), there was greater similarity with the actinobacteria group than the fungus group. These suggest a more pronounced antifungal activity for the ACTB-290 strain, which agrees with the SEM images of the fungus filaments after its inhibition by ACTB-290, as previously discussed.

The loadings graphs displayed in Figure 8c ( $\mathrm{PC} 1 v s$. $\mathrm{PC} 2)$ and Figure 8d (PC1 vs. PC3) show that mVOCs inside the yellow circle (Figure 8c) present significantly higher concentration for ACTB-77 samples when compared with the other groups, besides having higher loadings values in PC1. The mVOCs inside the blue circle present a significantly higher concentration of both ACTB-290 and the co-culture (ACTB-290/C. musae), indicating that these two groups present similar mVOCs.

Dimethyl disulfide (4), 2-undecanone (43) and 2-tridecanone (54), Table 2, are present in a significatively lower concentration in the co-culture ACTB-290/C. musae when compared with the axenic culture of the actinobacteria (Figures $8 \mathrm{c}$ and $8 \mathrm{~d}$ ). Ketones $\mathbf{4 3}$ and $\mathbf{5 4}$ are those produced exclusively by the actinobacteria, while dimethyl disulfide (4) is a potent fungicide.

A $t$-test was performed using PC2 scores produced no statistically significant differences between the ACTB-290 group and the $C$. musae/ACTB-290 group $\left(t=-0.3265 / t_{\text {crit }}=2.447\right.$ and $\left.p=0.7551\right)$. These groups have similar values of PC 2 scores, and the same occurs with PC1. This fact corroborates the result already discussed that the co-culture (C. musae/ACTB-290) is very similar to the axenic culture of the actinobacteria. However, when both $\mathrm{PC} 1$ and $\mathrm{PC} 2$ are used, the variance hypothesis test indicated statistical differences between them. This latter 

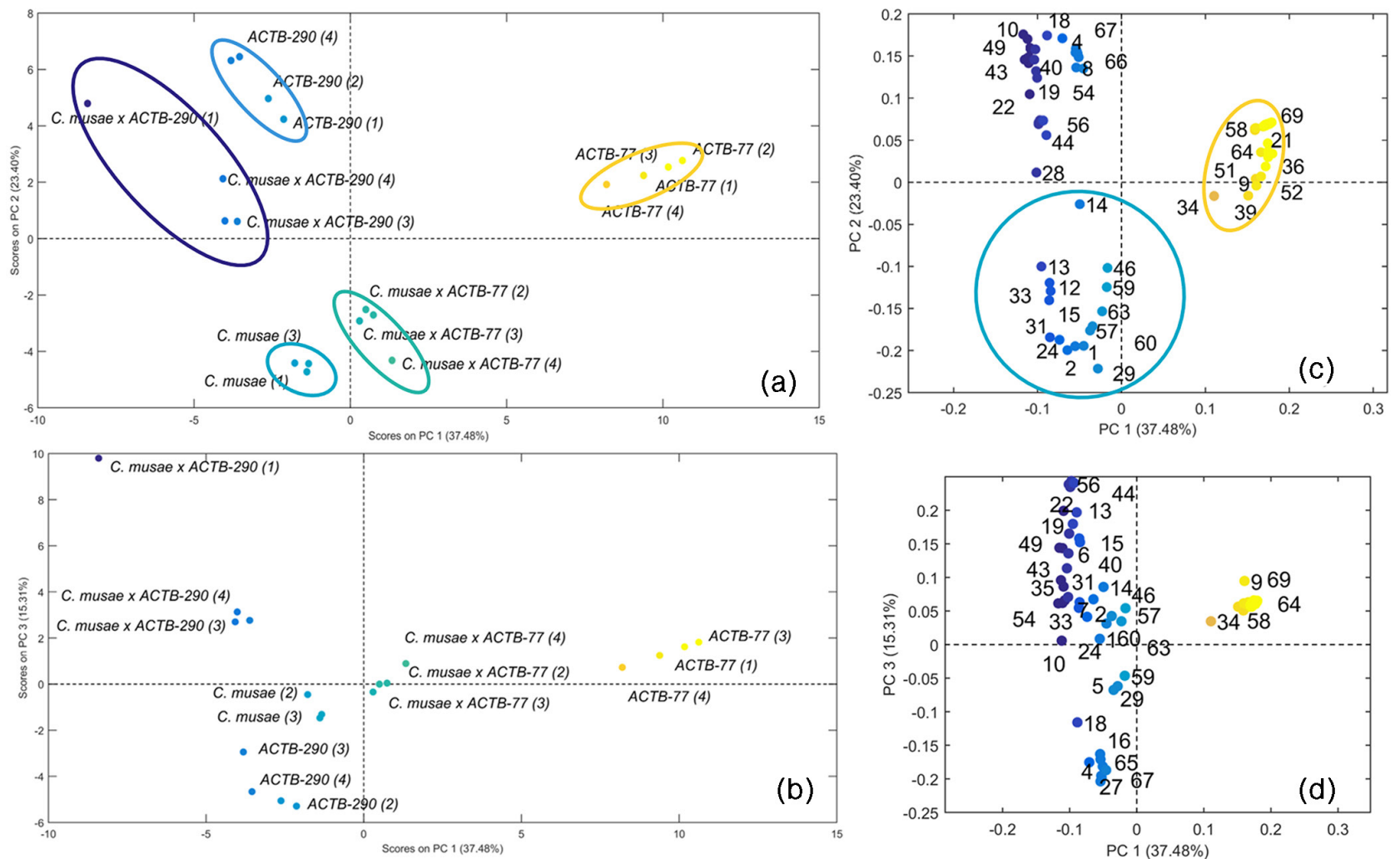

Figure 8. Scores (a) and loadings (b) graphs obtained by PC1 and PC2 for the samples ACTB-77 (yellow), ACTB-290 (blue), C. musae (cyan) and co-cultures ACTB-77/C. musae (light green) and ACTB-290/C. musae (dark blue and blue). Scores (c) and loadings (d) graphs obtained by PC1 and PC3 for the same samples.

approach was similar to the one used in Soft Independent Method of Class Analogy (SIMCA). ${ }^{43}$

A correlation map of $\mathrm{mVOCs}$ produced in all experiments is presented in Figure S7 (SI section), where each axis represents the constituents numbered according to Table 2 . The correlation is calculated pair by pair using the mVOCs, and represented by squares on the map: dark blue means an inverse correlation $(\mathrm{r}=$ $-1)$ and dark red means a direct correlation $(r=+1)$. The closer the correlation values (r) are to $+1 /-1$, the higher the correlation between the peaks will be. Analysis of this map reveals that the ketones 22 and $\mathbf{4 3}$, which were suggested as being reduced by the fungus, present an inverse correlation with their respective alcohols 23 and 44 (Table 2), confirming a biotransformation occurrence in the co-culture. In addition, there is a slightly positive correlation between these ketones and the sulfur-containing compound $\mathbf{4}$, suggesting that when the concentration of the ketones reduces, the concentration of $\mathbf{4}$ also reduces.

In summary, these analyses lead to the conclusion that the ACTB-290 strain presents a powerful and specific fungicide effect against $C$. musae. This gives rise to the hypothesis that the sulfur-containing constituents are responsible for their bioactivity.

\section{Conclusions}

In summary, rhizosphere soil of plants from the Caatinga biome was shown to be source of actinobacteria strains that produced volatile organic compounds (VOCs) with antifungal activity against the phytopathogen C. musae. Among the investigated strains, the most active were Streptomyces sp. (ACTB-77) and Amycolatopsis sp. (ACTB-290). The latter presented the highest inhibition of fungus growth, a behavior corroborated by the greatest damage of its VOCs to the fungal hyphae morphology. HS-SPME-GCMS analyses of VOCs produced by ACTB-77 and ACTB-290, revealed linalool and geosmin as major constituents for ACTB-77, and dimethyl disulfide and dimethyl trisulfide as major VOCs compounds produced by ACTB-290. No exclusive VOCs were observed in the coculture experiment involving ACTB-77, while co-culture with ACTB-290 yielded five new peaks, two of them (alcohols) suggested as products of ketone bioreduction by the fungus. Statistical analysis showed that co-culture ACTB-77/C. musae was the most similar to the fungus, while co-culture ACTB-290/C. musae showed greater similarity with the actinobacteria. The more pronounced antifungal activity of ACTB-290 was suggested that it was associated to its sulfur-containing metabolites, since 
this class of compounds is known as a potent antifungal. Additionally, linalool was suggested as responsible for the ACTB-77 activity. A preliminary version of this work was published as preprint. ${ }^{63}$

\section{Supplementary Information}

Supplementary data (figures representing experimental procedures, mass spectra of compounds 1-69 and correlation maps) are available free of charge at http://jbcs.sbq.org.br as PDF file.

\section{Acknowledgments}

The authors are thankful to the Coordenação de Aperfeiçoamento de Ensino Superior (CAPES) for the sponsorships of M. V. Brito (process: 88887.319063/2019-00) and M. S. Netto (process: $88887.479095 / 2020-00$ ), and financial support (Finance Code 001 - PROEX 23038.000509/2020-82, No. AUXPE: 1227/2020). M. C. F. de Oliveira (process: 310881/20200 ) and M. C. de Mattos (process: 306043/2018-1) thank to Conselho Nacional de Desenvolvimento Científico e Tecnológico $(\mathrm{CNPq})$ for their research sponsorships. L. Pinto thank the Brazilian funding agency Fundação de Amparo a Ciência e Tecnologia do Estado de Pernambuco (FACEPE 14/2019 - INOVA IAM; process: APQ-04371.06/19). The English text of this paper has been revised by Sidney Pratt, Canadian, MAT (The Johns Hopkins University), RSAdip - TESL (Cambridge University).

\section{Author Contributions}

All authors contributed to the study conception and design. Maria V. de Brito, Wéverson L. Fonseca, Jair Mafezoli, Francisco G. Barbosa, Fátima M. Nunes, Marcos C. de Mattos, João E. A. dos Santos and Maria C. F. Oliveira were responsible for culture of the microorganisms, VOCs extractions and analyses (HS-SPMEGCMS), actinobacteria molecular identification, manuscript writing and revision; Francisca S. A. Araujo, Regimara F. B. S Vieira, Hilton C. R. Magalhães, Celli R. Muniz and Deborah S. Garruti for SEM image analysis, GC-MS equipment operation and microorganisms preservation; Marcio A. Ootani and Francisco M. P. Viana for isolation of actinobacteria strains from rhizosphere associated to Caatinga plants and Colletotrichum musae from infected plant; José M. S. Netto and Licarion Pinto for statistical analyses. The first draft of the manuscript was written by Maria $\mathrm{C}$. F. Oliveira (corresponding author) and all authors commented on previous versions of the manuscript. All authors read and approved the final manuscript.

\section{References}

1. Costa, A. C.; Miranda, R. F.; Costa, F. A.; Ulhoa, C. J.; Biocatal. Agric. Biotechnol. 2021, 34, 102.

2. Vilaplana, R.; Pazmiño, L.; Valencia-Chamorro, S.; Postharvest Biol Technol. 2018, 138, 56.

3. Damasceno, C. L.; Duarte, E. A. A.; Santos, L. B. P. R.; Oliveira, T. A. S.; Jesus, F. N.; Oliveira, L. M.; Góes-Neto, A.; Soares, A. C. F.; Biol. Control 2019, 137, 104.

4. Harir, M.; Bendif, H.; Bellahcene, M.; Fortas, Z.; Pogni, R. In Basic Biology and Applications of Actinobacteria; Enany, S., ed.; IntechOpen, 2018, ch. 6, DOI: 10.5772/intechopen.79890.

5. David, B.; Wolfender, J. L.; Dias, D. A.; Phytochem. Rev. 2015, 14, 299.

6. Schulz-Bohm, K.; Martín-Sánchez, L.; Garbeva, P.; Front. Microbiol. 2017, 8, 24.

7. Mari, M.; Bautista-Baños, S.; Sivakumar, D.; Postharvest Biol. Technol. 2016, 122, 70

8. Choudoir, M.; Rossabi, S.; Gebert, M.; Helmig, D.; Fierer, N.; mSystems 2019, 4, e00295-18.

9. Ross, C.; Opel, V.; Scherlach, K.; Hertweck, C.; Mycoses 2014, $57,48$.

10. Brakhage, A. A.; Schoroeckh, V.; Fungal Genet. Biol. 2011, 48,15 .

11. Bertrand, S.; Bohni, N.; Schnee, S.; Schumpp, O.; Gindro, K.; Wolfender, J. L.; Biotechnol. Adv. 2014, 32, 1180.

12. Bertrand, S.; Schumpp, O.; Bohni, N.; Monod, M.; Gindro, K.; Wolfender, J. L.; J. Nat. Prod. 2013, 76, 1157.

13. Deng, X.; Zhang, N.; Shen, Z.; Zhu, C.; Li, R.; Salles, J. F.; Shen, Q.; Appl. Soil Ecol. 2020, 147, 103364.

14. Bonfante, P.; Anca, I. A.; Annu. Rev. Microbiol. 2009, 63, 363.

15. Yadav, A. K.; Srivastava, A. K.; Yandigeri, M. S.; Kashyap, S. K.; Modi, D. R.; Arora, D. K.; Ann. Microbiol. 2010, 60, 605.

16. Sharma, M.; Dangi, P.; Choudhary, M.; Int. J. Curr. Microbiol. Appl. Sci. 2014, 3, 801.

17. Scholler, C. E. G.; Gurtler, H.; Pedersen, R.; Molin, S.; Wilkins, K.; J. Agric. Food Chem. 2002, 50, 2615.

18. Sharma, V.; Salwan, R. In New and Future Developments in Microbial Biotechnology and Bioengineering; Singh, J. S., ed.; Elsevier: India, 2018, ch. 6.

19. Boukaew, S.; Plubrukam, A.; Prasertsan, P.; BioControl 2013, 58,471 .

20. Li, Q.; Ning, P.; Zheng, L.; Huang, J.; Li, G.; Hsiang, T.; Biol. Control 2012, 61, 113.

21. Nzekoue, F. K.; Caprioli, G.; Fiorini, D.; Torregiani, E.; Vittori, S.; Sagratin, G.; Food Res. Int. 2019, 12, 730.

22. Maia, R.; Correia, M.; Pereira, I. M. B.; Beleza, V. M.; Microchem. J. 2014, 112, 164.

23. Gherghel, S.; Morgan, R. M.; Arrebola-Liébanas, J.; RomeroGonzález, R.; Blackman, C. S.; Garrido-Frenich, A.; Forensic Sci. 2018, 290, 207. 
24. Hantao, L. W.; Aleme, H. G.; Passador, M. M.; Furtado, E. L.; Ribeiro, F. A. L.; Poppi, R. J.; Augusto, F.; J. Chromatogr. A 2013, 1279, 86.

25. Oliveira, F. C.; Barbosa, F. G.; Mafezoli, J.; Oliveira, M. C. F.; Camelo, A. L. M.; Longhinotti, E.; Lima, A. C. A.; Câmara, M. P. S.; Gonçalves, F. J. T.; Freire, F. C. O.; J. Braz. Chem. Soc. 2015, 26, 2189.

26. Lazazzara, V.; Perazzolli, M.; Pertot, I.; Biasioli, F.; Puopolo, G.; Cappellin, L.; Microbiol. Res. 2017, 201, 52.

27. Amini, J.; Agapoor, Z.; Ashengroph, M.; J. Plant Prot. Res. 2016, 56, 254.

28. Motulsky, H.; GraphPad Prism 6.01; University of California San Diego, California, 1989.

29. Zhang, Q.; Zhang, J.; Yang, L.; Zhang, L.; Jiang, D.; Chen, W.; Li, G.; Biol. Control 2014, 72, 98.

30. Kumar, S.; Stecher, G.; Tamura, K.; Mol. Biol. Evol. 2016, 33, 1870.

31. Hall, T. A.; Nucleic Acids Symp. Ser. 1999, 41, 95.

32. GenBank Overview; https://blast.ncbi.nlm.nih.gov/ Blast.cgi?PAGE_TYPE=BlastSearch\&BLAST SPEC=MicrobialGenomes, accessed in February 2022.

33. Higgins, D.; Sievers, F.; Dineen, D.; Wilm, A.; ClustalW 2.0.12; Bioinformatics Institute Cambridge, United Kingdom, 1994.

34. Swofford, D. 1.; PAUP 4.0 program beta 10; University of Massachusetts, United States, 2002.

35. Asahi, Y.; Miura, J.; Tsuda, T.; Kuwabata, S.; Tsunashima, K.; Noiri, Y.; Sakata, T.; Ebisu, S.; Hayashi, M.; AMB Express 2015, 5,6 .

36. Sawoszczuk, T.; Sygula-Cholewinska, J.; Hoyo-Meléndez, J. M. D.; J. Chromatogr. A 2015, 1409, 30.

37. Chambers, J. M.; R Programming language; Lucent Technologies, Vienna, Austria, 1993.

38. Adams, R. P.; Identification of Essential Oil Components by Gas Chromatography/Mass Spectrometry, $4^{\text {th }}$ ed.; Carol Stream: Illinois, USA, 2017.

39. NIST Livro de Química na Web; https://webbook.nist.gov/cgi/ cbook.cgi?Name $=$ Geosmin $\&$ Units $=$ SI, accessed in February 2022.

40. PubChem; https://pubchem.ncbi.nlm.nih.gov/\#query=Linalool, accessed in February 2022.

41. Wise, B. M.; Gallagher N. B.; Bro, R.; Shaver, J. M.; Windig, W.; Scott, R.; KochPLS Toolbox version 5.2 for use with MATLAB ${ }^{\mathrm{TM}}$, Eigenvector Research, Wenatchee, 2009.

42. MATLAB, version 7.10.0 (R2010a); Natick, The MathWorks Inc., Massachusetts, USA, 2010.

43. Brereton, R. G.; J. Chemom. 2011, 25, 225.

44. Couto, E. F.; Menezes, M.; Fitopatol. Bras. 2005, 29, 406.

45. Jayakumar, V.; Ramesh, S. A.; Viswanathan, R.; Sugar Tech. 2021, 23, 94.
46. Zhao, P.; Li, P.; Wu, S.; Zhou, M.; Zhi, R.; Gao, H.; AMB Express 2019, 9, 119.

47. Quintana-Rodriguez, E.; Morales-Vargas, A. T.; Molina-Torres, J.; Ádame-Alvarez, R. M.; Acosta-Gallegos, J. A.; Heil, M.; J. Ecol. 2014, 103, 250.

48. Rojas-Flores, C.; Ventura-Aguilar, R. I.; Bautista-Baños, S.; Revah, S.; Saucedo-Lucero, J. O.; Microbiol. Res. 2019, 228, 2.

49. Cordovez, V.; Carrion, V. J.; Etalo, D. W.; Mumm, R.; Zhu, H.; Wezil, G. P. V.; Raaijmakers, J. M.; Front. Microbiol. 2015, 6, 1081.

50. Boukaew, S.; Prasertsan, P.; J. Appl. Microbiol. 2020, 129, 652.

51. Karuppiah, V.; Ranaghan, K. E.; Leferink, N. G. H.; Johannissen, L. O.; Shanmugam, M.; Cheallaigh, A. N.; Bennett, N. J.; Kearsey, L. J.; Takano, E.; Gardiner, J. M.; van der Kamp, M. W.; Hay, S.; Mulholland, A. J.; Leys, D.; Scrutton, N. S.; ACS Catalysis 2017, 7, 6268.

52. Zhao, P.; Xue, Y.; Gao, W.; Li, J.; Zu, X.; Fu, D.; Feng, S.; Bai, X.; Zuo, Y.; Li, P.; Peptides 2018, 103, 48.

53. Lan-Phi, N. T.; Vy, T. T.; Int. Food Res. 2015, 22, 2426.

54. Mohammadhosseini, M.; Asian J. Chem. 2012, 24, 3814.

55. Oliveira, L. M. I.; Araújo, M. A. C.; Souza, S. K. V.; Cardoso, G. N.; Oliveira, L. E.; Oliveira, P. F.; J. Mycol. Med. 2017, 27, 195.

56. Dias, I. J.; Trajano, E.; Castro, R. D.; Ferreira, G. L. S.; Medeiros, H. C. M.; Gomes, D. Q. C.; Braz. J. Biol. 2017, 78, 368.

57. Zhou, H.; Tao, N.; Jia, L.; Food Control 2014, 37, 277.

58. Lira, M. H. P.; Andrade Jr., F. P.; Moraes, G. F. Q.; Macena, G. S.; Pereira, F. O.; Lima, I. O.; J. Essent. Oil Res. 2020, 32, 187.

59. Elshafie, H. S.; Mancini, E.; Sakr, S.; de Martino, L.; Mattia, C. A.; de Feo, V.; Camele, I.; J. Med. Food 2015, 18, 929.

60. Wang, C.; Wang, Z.; Qiao, X.; Li, Z.; Li, F.; Chen, M.; Wang, Y.; Huang, Y.; Cui, H.; FEMS Microbiol. Lett. 2013, 341, 45.

61. Wang, Z.; Zhong, T.; Chen, K.; Du, M.; Chen, G.; Chen, X.; Wang, K.; Zalan, Z.; Takacs, K.; Kan, J.; Food Control 2021, 120, 107499.

62. Bro, R.; Smilde A. K.; Anal. Methods 2014, 6, 2812.

63. de Brito, M. V.; Fonseca, W. L.; Mafezoli, J.; Barbosa, F. G.; Nunes, F. M.; de Mattos, M. C.; dos Santos, J. E. A.; Araujo, F. S. A.; Vieira, R. F. B. S.; Magalhães, H. C. R.; Muniz, C. R.; Garruti, D. S.; Ootani, M. A.; Netto, J. M. S.; Pinto, L.; Viana, F. M. P.; de Oliveira, M. C. F.; ResearchSquare, 2021, DOI: 10.21203/rs.3.rs-509649/v1, available at https://www. researchsquare.com/article/rs-509649/v1, accessed in February 2022.

Submitted: September 23, 2021 Published online: February 23, 2022 